\title{
R-parity conservation via the Stueckelberg mechanism: LHC and Dark Matter Signals
}

\author{
Daniel Feldman, ${ }^{a, b}$ Pavel Fileviez Perez $^{c}$ and Pran Nath ${ }^{d}$ \\ ${ }^{a}$ Michigan Center for Theoretical Physics, University of Michigan, \\ Ann Arbor, $M I$ 48109, U.S.A. \\ ${ }^{b}$ CERN Theory Group Visitor, \\ CH-1211 Geneva 23, Switzerland \\ ${ }^{c}$ Center for Cosmology and Particle Physics (CCPP), Department of Physics, \\ New York University, NY 10003, New York, U.S.A. \\ ${ }^{d}$ Northeastern University, Department of Physics, \\ Boston, MA 02115, U.S.A. \\ E-mail: djfeld@umich.edu, pfp1@nyu.edu, nath@neu.edu
}

ABSTRACT: We investigate the connection between the conservation of R-parity in supersymmetry and the Stueckelberg mechanism for the mass generation of the $B-L$ vector gauge boson. It is shown that with universal boundary conditions for soft terms of sfermions in each family at the high scale and with the Stueckelberg mechanism for generating mass for the $B-L$ gauge boson present in the theory, electric charge conservation guarantees the conservation of R-parity in the minimal $B-L$ extended supersymmetric standard model. We also discuss non-minimal extensions. This includes extensions where the gauge symmetries arise with an additional $\mathrm{U}(1)_{B-L} \otimes \mathrm{U}(1)_{X}$, where $\mathrm{U}(1)_{X}$ is a hidden sector gauge group. In this case the presence of the additional $\mathrm{U}(1)_{X}$ allows for a $Z^{\prime}$ gauge boson mass with $B-L$ interactions to lie in the sub-TeV region overcoming the multi- $\mathrm{TeV}$ LEP constraints. The possible tests of the models at colliders and in dark matter experiments are analyzed including signals of a low mass $Z^{\prime}$ resonance and the production of spin zero bosons and their decays into two photons. In this model two types of dark matter candidates emerge which are Majorana and Dirac particles. Predictions are made for a possible simultaneous observation of new physics events in dark matter experiments and at the LHC.

Keywords: Supersymmetric gauge theory, Beyond Standard Model, Discrete and Finite Symmetries

ARXIV EPRINT: 1109.2901 


\section{Contents}

1 Introduction 1

$2 \quad B-L$ Stueckelberg extension of the standard model $\quad 3$

$3 \quad B-L$ Stueckelberg extension of the MSSM $\quad 4$

4 R-parity conservation $\quad \mathbf{5}$

4.1 Scalar potential and R-parity conservation 5

$5 \quad \mathrm{U}(1)_{B-L} \otimes \mathrm{U}(1)_{X}$ Stueckelberg model $\quad 8$

5.1 Production of vector resonances 11

$\begin{array}{lll}5.2 & \text { Production and decay of the scalars } \rho \text { and } \rho^{\prime} & 12\end{array}$

6 Neutral Dirac and Majorana components of dark matter $\quad 17$

$\begin{array}{ll}6.1 \text { Majorana dark matter } & 17\end{array}$

$\begin{array}{ll}6.2 \text { Dirac dark matter } & 19\end{array}$

7 Discriminating Stueckelberg from models with spontaneous breaking $\quad 24$

7.1 Spontaneous symmetry breaking of $B-L$ and R-parity violation 24

$\begin{array}{lll}7.2 & B-L \text { models for R-parity conservation } & 24\end{array}$

8 Conclusion $\quad 25$

\section{Introduction}

R-parity is an important symmetry in supersymmetric theories (For a review see [1]). In supergravity theories [2-5], over most of the parameter space of models consistent with the radiative breaking of the electroweak symmetry, the lightest neutralino is found to be the lightest supersymmetric particle, and this, along with R-parity (defined as $R=$ $(-1)^{2 S+3(B-L)}$, where $S, B$ and $L$ stand for the spin, baryon number and lepton number, respectively) and charge neutrality allows for the lightest neutralino to be a promising candidate for cold dark matter as suggested in [6].

The question then, is, if indeed R-parity turns out to be a conserved symmetry of nature, how does such a symmetry come about, and how one may guarantee that it is conserved. It is known that the MSSM with the inclusion of a right handed neutrino, one for each generation, has an anomaly free $\mathrm{U}(1)_{B-L}$ which can be gauged. ${ }^{1}$ Of course, a $\mathrm{U}(1)_{B-L}$ gauge boson must grow mass otherwise it would produce an undesirable long range force. In the analysis that follows it is shown that a gauged $B-L$ symmetry, where the

\footnotetext{
${ }^{1} \mathrm{~A}$ gauged $\mathrm{U}(1)_{B-L}$ arises naturally in GUT models such as $\mathrm{SO}(10)$ and $E_{6}$ and in string models.
} 
gauge boson develops a mass through the Stueckelberg mechanism extending the Standard Model gauge group [7-11] preserves R-parity, i.e., R-parity does not undergo spontaneous breaking by renormalization groups effects under the assumption of universality of soft scalar masses, charge conservation and in the absence of a Fayet-Iliopoulos D-term. We will later refer to this model as the Minimal B - L Stueckelberg Extension of the MSSM.

The fact that the minimal gauged $B-L$ model proposed in this work preserves Rparity, with mass growth arising from the Stueckelberg mechanism, is in contrast to models with a gauged $B-L$ where the symmetry is broken spontaneously and thus does not necessarily preserve the R-parity invariance. Thus the analyses of [12-17] show that R-parity symmetry, even if valid at the grand unification scale, could be broken by renormalization group effects. ${ }^{2}$

We will first discuss the minimal $(B-L)$ Stueckelberg extension of the Standard Model and of the minimal supersymmetric Standard Model (MSSM). In these extensions the $Z^{\prime}$ boson $^{3}$ is constrained to be rather heavy, i.e., it lies in the multi-TeV range and thus a direct detection may be difficult. However, this constraint is overcome in a $\mathrm{U}(1)_{B-L} \otimes$ $\mathrm{U}(1)_{X}$ Stueckelberg extension, where $\mathrm{U}(1)_{X}$ is the hidden sector gauge group. Here the Stueckelberg sector generates two extra massive vector neutral bosons, i.e., $Z^{\prime}$ and $Z^{\prime \prime}$, one of which would be very narrow and could lie even in the sub-TeV region, and thus would be accessible at the LHC. The models with massive mediators arise generally via mass mixing and kinetic mixing of Abelian gauge bosons ([30-43]; for additional works, see [44, Chapter 8, pg. 136]) and the mixings are also the source of the so called dark forces [30, 32] — the mixings allow for a portal between the hidden (dark) sector via massive mediators [30-36] (from which several components of dark matter can arise) and the visible sector where the states charged under the the Standard Model reside. Specifically, the class of models that we study here allows for two component (Majorana and Dirac) dark matter [45]. Such models with dark forces have received considerable attention in the context of the recent cosmic anomalies [45-51]; for recent additional works on dark sectors see e.g. [52-59].

The organization of this paper is as follows: in section 2 we propose a $\mathrm{U}(1)_{B-L}$ extension of the Standard Model via the Stueckelberg mechanism. In section 3 the $B-L$ Stueckelberg extension of MSSM is introduced. In section 4 we outline the conditions for R-parity to be not spontaneously broken. In section 5 we give a dedicated analysis of a $\mathrm{U}(1)_{B-L} \otimes \mathrm{U}(1)_{X}$ extension of the MSSM via the Stueckelberg mechanism and show that the model naturally leads to a sharp $Z^{\prime}$ prime resonance that can be seen at the LHC, and we analyze recent constraints from the Tevatron and the LHC. Here we also analyze the production and decay of new spin-0 particles. These scalars are the real parts of the chiral Stueckelberg superfields, where the imaginary part are the axions which are absorbed giving masses to the $Z^{\prime}$ and $Z^{\prime \prime}$. In section 6 we show that the model allows for two component dark matter, one consisting of neutral Dirac dark matter and the other of Majorana dark matter which produce a relic abundance consistent with WMAP [60]. We also explore the

\footnotetext{
${ }^{2}$ For grand unified models where R-parity symmetry is automatic see [18]. For analyses where the spontaneous breaking of $B-L$ occurs see [19, 20], for early work on the spontaneous breaking of R-parity see [21-24] . For early analyses with R-parity and additional gauge fields see [25].

${ }^{3}$ For recent dedicated work on heavy $Z_{B-L}^{\prime}$ physics see [26-29].
} 
detection possibility of dark matter with the recent limits set by the XENON and CDMS collaborations $[61,62]$ which allows for direct detection constraints to be connected with the corresponding constraints on the $Z^{\prime}$ production at colliders. In section 7 we give an overview as to how models of spontaneous R-parity breaking can be distinguished from the R-parity preserving $B-L$ extensions. Conclusions are given in section 8 .

\section{$2 \quad B-L$ Stueckelberg extension of the standard model}

The $B-L$ extension of the Standard Model provides a natural framework to understand the origin of neutrino masses since the three families of right-handed neutrinos, needed to cancel all anomalies, are used to generate neutrino masses. We first consider a $\mathrm{U}(1)_{B-L}$ Stueckelberg extension of the Standard Model with the gauge group

$$
\mathrm{SU}(3)_{C} \otimes \mathrm{SU}(2)_{L} \otimes \mathrm{U}(1)_{Y} \otimes \mathrm{U}(1)_{B-L}
$$

The mass growth for the $\mathrm{U}(1)_{B-L}$ occurs via the Stueckelberg mechanism for which the extended Lagrangian is given by

$$
\begin{aligned}
\mathcal{L} & =\mathcal{L}_{\mathrm{St}}^{B-L}+\mathcal{L}_{\text {Yuk }}^{B-L}+\mathcal{L}_{\mathrm{SM}}, \\
\mathcal{L}_{\mathrm{St}}^{B-L} & =-\frac{1}{4} C_{\mu \nu} C^{\mu \nu}-\frac{1}{2}\left(M_{\mathrm{BL}} C_{\mu}+\partial_{\mu} \sigma\right)\left(M_{\mathrm{BL}} C^{\mu}+\partial^{\mu} \sigma\right), \\
\mathcal{L}_{\text {Yuk }}^{B-L} & =Y_{\nu} \bar{l}_{L} \tilde{H} \nu_{R} .
\end{aligned}
$$

Here $\mathcal{L}_{\mathrm{SM}}$ is the Standard Model Lagrangian, $l_{L}^{T}=\left(\nu_{L}, e_{L}\right)$ and $\tilde{H}=i \sigma_{2} H^{*}$. As usual, the Standard Model Higgs is $H^{T}=\left(H^{+}, H^{0}\right)$. The above Lagrangian is invariant under the $B-L$ transformations

$$
\delta C_{\mu}=\partial_{\mu} \lambda, \quad \delta \sigma=-M_{\mathrm{BL}} \lambda .
$$

Added to the above is a gauge fixing term

$$
\mathcal{L}_{\mathrm{gf}}=-\frac{1}{2 \xi}\left(\partial_{\mu} C^{\mu}+M_{\mathrm{BL}} \xi \sigma\right)^{2},
$$

so that the vector field becomes massive while the $\sigma$ field decouples. Additionally the interaction Lagrangian

$$
\mathcal{L}_{\mathrm{St}}^{\mathrm{int}}=g_{\mathrm{BL}} C_{\mu} J_{\mathrm{BL}}^{\mu}
$$

couples the Stueckelberg field $C_{\mu}$ to the conserved $B-L$ vector current $J_{\mathrm{BL}}^{\mu}$. We note that the $B-L$ gauge field $C_{\mu}$ has become massive with a mass $M_{\mathrm{BL}}$ while maintaining the $\mathrm{U}(1)_{B-L}$ invariance. Since $B-L$ continues to be a symmetry even after the mass growth of the $Z^{\prime}$ its properties are rather different from the model where the $B-L$ gauge symmetry is spontaneously broken through the Higgs mechanism. We will return to this in a later section. It is important to mention that in this theory the neutrinos are Dirac fermions since there is no way to generate Majorana masses for right-handed neutrinos as in the canonical $B-L$ model. This is a natural consequence coming from the Stueckelberg mechanism. In the above we have assumed that the $B-L$ scale is in the $\mathrm{TeV}$ region. 
Here our choice is at the same level as for the extra dimension models and for the warped extra-dimension models, i.e., this scale is interesting because it is related to the electroweak physics which is being explored at the LHC.

In the above, a kinetic mixing term is possible leading to a generalized mass and kinetic mixings for a massive $\mathrm{U}(1)$ which will then generally mix with the SM sector [30, 32] where the hypercharge vector boson $B$ mixes via both mass and kinetic mixings [30]. One then diagonalizes the Stueckelberg mass and kinetic mixing together [32, 63-67]. A further generalization to multiple $\mathrm{U}(1) s$ reads

$$
\mathcal{L}_{\mathrm{St}}^{\mathrm{KM}}=\frac{1}{2} \sum_{i, j, i \neq j}^{N_{V}} \frac{\epsilon_{i j}}{2} V_{i \mu \nu} V_{j}^{\mu \nu}-\frac{1}{2} \sum_{n=1}^{N_{S}}\left(\partial_{\mu} \sigma_{n}+\sum_{m=1}^{N_{V}} M_{n m} V_{\mu m}\right)^{2},
$$

with $N_{V}$ Abelian vectors and $N_{S}$ axions, where $B_{\mu}=V_{\mu 1}$ and the other vector fields correspond to either hidden or visible gauge symmetries. Recent works with multiple additional $\mathrm{U}(1) s$ have indeed been discussed recently [32, 45, 48-52, 68]. Our analysis is restricted to non-anomalous extension of the Standard Model (for the anomalous case see e.g. [69-74]). In the analysis that follows we will assume the kinetic mixing is absent and instead investigate the pure Stueckelberg sector in the absence of mass mixing of the hypercharge $B$ with the Stueckelberg sector. For recent works on the Stueckelberg Mechanism see e.g. [53-56, 75-78] and for early work in the context of strings see [79].

\section{$3 \quad B-L$ Stueckelberg extension of the MSSM}

Here we construct the minimal $\mathrm{U}(1)_{B-L}$ extension of the MSSM using the Stueckelberg Mechanism. The supersymmetric extension of eq. (2.4) is

$$
\mathcal{L}_{\mathrm{St}}=\left.\left(M_{\mathrm{BL}} C+S_{\mathrm{st}}+\bar{S}_{\mathrm{st}}\right)^{2}\right|_{\theta^{2} \bar{\theta}^{2}},
$$

where $C=\left(C_{\mu}, \lambda_{C}, D_{C}\right)$ is the gauge vector multiplet for $\mathrm{U}(1)_{B-L}$, and the Stueckelberg multiplet is $S_{\mathrm{st}}=\left(\rho+i \sigma, \psi_{\mathrm{st}}, F_{S}\right)$ where $\rho$ is a scalar while $\sigma$ is the axionic pseudo-scalar. The supersymmetrized gauge transformations under the $\mathrm{U}(1)_{B-L}$ are

$$
\delta_{\mathrm{BL}} C=\zeta_{\mathrm{BL}}+\bar{\zeta}_{\mathrm{BL}}, \quad \delta_{\mathrm{BL}} S_{\mathrm{st}}=-M_{\mathrm{BL}} \zeta_{\mathrm{BL}}
$$

where $\zeta$ is an infinitesimal chiral superfield. Next we couple the chiral matter fields $\Phi_{i}$ consisting of quarks, leptons and Higgs fields of MSSM. These couplings are given by

$$
\mathcal{L}_{\text {matter }}=\left.\bar{\Phi}_{m} e^{2 g_{\mathrm{BL}} Q_{\mathrm{BL}} C} \Phi_{m}\right|_{\theta^{2} \bar{\theta}^{2}}
$$

where $Q_{\mathrm{BL}} \equiv B-L$ and the sum is implicit over the chiral multiplets $m$ and the interaction term of eq. (2.7) couples the $B-L$ vector field to fermions. We focus on the bosonic part of the extended Lagrangian which is given by

$$
\begin{aligned}
\mathcal{L}_{\text {spin }[0,1]}= & -\frac{1}{4} C_{\mu \nu} C^{\mu \nu}-\frac{1}{2} M_{\mathrm{BL}}^{2} C_{\mu}^{2}-\frac{1}{2}\left(\partial_{\mu} \rho\right)^{2}-\frac{1}{2} M_{\mathrm{BL}}^{2} \rho^{2} \\
& -\left|D_{\mu} \tilde{f}_{i}\right|^{2}-g_{\mathrm{BL}} M_{\mathrm{BL}} \rho \tilde{f}_{i}^{\dagger} Q_{B L} \tilde{f}_{i}-\frac{1}{2}\left[\sum_{i} \tilde{f}_{i}^{\dagger} g_{\mathrm{BL}} Q_{B L} \tilde{f}_{i}\right]^{2} .
\end{aligned}
$$


The superpotential of the $B-L$ extended theory is simply

$$
\mathcal{W}=\mu H_{u} H_{d}+\sum_{\text {gen }}\left[Y_{u} Q H_{u} u^{c}+Y_{d} Q H_{d} d^{c}+Y_{e} L H_{d} e^{c}+Y_{\nu} L H_{u} \nu^{c}\right]
$$

Aside from the term $Y_{\nu} L H_{u} \nu^{c}$ eq. (3.5) is the superpotential of MSSM but without the terms that violate R-parity.

\section{R-parity conservation}

As pointed out earlier, while the Stueckelberg mechanism gives mass to the $B-L$ gauge boson, the Lagrangian of the theory, after the mass growth, still has a $B-L$ symmetry and hence a conservation of R-parity $\left(R=(-1)^{2 S+3(B-L)}=(-1)^{2 S} M\right.$. Here $M$ denotes matter parity, which is +1 for Higgs and gauge superfields, and -1 for all matter chiral superfields). This conservation of R-parity in the minimal $B-L$ Stueckelberg extensions is in contrast to models where the $B-L$ gauge symmetry is broken by a Higgs mechanism and where in general the mass growth of the $B-L$ gauge boson could break the $B-L$ symmetry and thus R-parity invariance is also lost. For example, for the model of eq. (3.5), a VEV growth for the scalar field in the $\nu_{l}^{c}$ multiplet will break $B-L$ invariance and generate a mass for the $B-L$ gauge boson. However, a VEV growth for $\tilde{\nu}_{l}^{c}$ also violates R-parity invariance which then removes the neutralino as a possible candidate for dark matter. Specifically, for example, in eq. (3.5) the VEV growth of $\tilde{\nu}_{l}^{c}$ generates the term $L H_{u}$ which breaks R-parity. However, in the minimal $B-L$ Stueckelberg extension of MSSM even after the mass growth of the $B-L$ gauge boson R-parity is maintained and the R-parity violating interactions such as $L H_{u}, L L e^{c}, Q L d^{c}, u^{c} d^{c} d^{c}$ are all forbidden in the superpotential.

\subsection{Scalar potential and R-parity conservation}

We wish to show here that with a Stueckelberg mechanism for mass generation the $B-L$ symmetry not only remains unbroken at the tree level but further that this invariance is not violated by radiative breaking in the minimal model. We give now the deduction of this result which is rather straightforward. We exhibit below the potential including just one generation of leptonic scalar fields in the model consisting of $\rho, \tilde{\nu}, \tilde{e}, \tilde{e}^{c}, \tilde{\nu}^{c}$ (An extension to 3 generations is trivial). Assuming charge conservation so that $\langle\tilde{e}\rangle=0=\left\langle\tilde{e}^{c}\right\rangle$, etc., and including soft breaking, the potential that involves $\rho, \tilde{\nu}$ and $\tilde{\nu}^{c}$ fields is

$$
\begin{aligned}
V_{\mathrm{St}-B L}= & \frac{1}{2}\left(M_{\mathrm{BL}}^{2}+m_{\rho}^{2}\right) \rho^{2}+M_{\tilde{\nu}}^{2} \tilde{\nu}^{\dagger} \tilde{\nu}+M_{\tilde{\nu}^{c}}^{2} \tilde{\nu}^{c \dagger} \tilde{\nu}^{c} \\
& +\frac{g_{\mathrm{BL}}^{2}}{2}\left(\tilde{\nu}^{c \dagger} \tilde{\nu}^{c}-\tilde{\nu}^{\dagger} \tilde{\nu}\right)^{2}+g_{\mathrm{BL}} M_{\mathrm{BL}} \rho\left(\tilde{\nu}^{c \dagger} \tilde{\nu}^{c}-\tilde{\nu}^{\dagger} \tilde{\nu}\right) \\
& +\left|Y_{\nu}\right|^{2}\left(\left|H_{u}^{0} \tilde{\nu}^{c}\right|^{2}+\left|\tilde{\nu} H_{u}^{0}\right|^{2}+\left|\tilde{\nu} \tilde{\nu}^{c}\right|^{2}\right) \\
& -\frac{1}{4}\left(g^{2}+g^{\prime 2}\right)\left(\left|H_{u}^{0}\right|^{2}-\left|H_{d}^{0}\right|^{2}\right)|\tilde{\nu}|^{2}+\frac{1}{8}\left(g^{2}+g^{\prime 2}\right)|\tilde{\nu}|^{4} \\
& +\left(-\mu^{*} Y_{\nu} H_{d}^{0 *} \tilde{\nu} \tilde{\nu}^{c}+A_{\nu} Y_{\nu} \tilde{\nu} \tilde{\nu}^{c} H_{u}^{0}+h . c .\right)
\end{aligned}
$$


where we have used $Q_{\mathrm{BL}}(e)=Q_{\mathrm{BL}}(\nu)=-1$ and where $m_{\rho}, M_{\tilde{\nu}}$, and $M_{\tilde{\nu}^{c}}$ are soft masses. The relevant part of the potential is then

$$
V=\sum_{\mathrm{gen}} V_{\mathrm{St}-B L}+V_{\mathrm{MSSM}}
$$

and where as is familiar

$$
\begin{aligned}
V_{\mathrm{MSSM}}= & \left(|\mu|^{2}+m_{H_{u}}^{2}\right)\left|H_{u}^{0}\right|^{2}+\left(|\mu|^{2}+m_{H_{d}}^{2}\right)\left|H_{d}^{0}\right|^{2}-\left(B \mu H_{u}^{0} H_{d}^{0}+\text { h.c. }\right) \\
& +\frac{1}{8}\left(g^{2}+g^{\prime 2}\right)\left(\left|H_{u}^{0}\right|^{2}-\left|H_{d}^{0}\right|^{2}\right)^{2} .
\end{aligned}
$$

We begin with universal boundary conditions for the RGEs. We note that the RG evolution for $M_{\tilde{e}}$ and $M_{\tilde{\nu}}$ are identical since $\mathrm{SU}(2)_{L} \otimes \mathrm{U}(1)_{Y}$ symmetry is unbroken down to electroweak scale. If $M_{\tilde{e}}^{2}$ turned tachyonic it would lead to VEV formation for the field $\tilde{e}$ violating charge conservation and thus we disallow this possibility. Since $\tilde{\nu}$ and $\tilde{e}$ lie in the same $\mathrm{SU}(2)_{L}$ multiplet the same holds for the $\tilde{\nu}$ field, i.e., it too does not develop a VEV. This can be seen from the one loop RG sum rule connecting the sneutrino $\tilde{\nu}$ mass and the selectron mass

$$
M_{\tilde{\nu}}^{2}-M_{\tilde{e}}^{2}=\cos (2 \beta) M_{W}^{2}+\delta_{\nu, e}^{2},
$$

where $\delta_{\nu, e}^{2}$ is difference of the mass squares of the fermions (and is essentially negligible compared to $W$ mass term the largest of which occurs for $e \rightarrow \tau$ which is still negligible). Thus the right hand side of eq. (4.4) is positive definite for any range of $\tan \beta$ in the perturbative domain in the RG analysis. As a consequence, if the mass square of $\tilde{e}$ does not turn tachyonic, this also holds for the mass square of $\tilde{\nu}$ and $\langle\tilde{\nu}\rangle=0$. Thus with $\langle\tilde{e}\rangle=0=\langle\tilde{\nu}\rangle=\left\langle\tilde{e}^{c}\right\rangle$, and integrating on the $\rho$ field, we get the following potential for $\tilde{\nu}^{c}$

$$
V_{\tilde{\nu}^{c}}=M_{\tilde{\nu}^{c}}^{2} \tilde{\nu}^{c \dagger} \tilde{\nu}^{c}+\frac{g_{\mathrm{BL}}^{2} m_{\rho}^{2}}{2\left(M_{\mathrm{BL}}^{2}+m_{\rho}^{2}\right)}\left(\tilde{\nu}^{c \dagger} \tilde{\nu}^{c}\right)^{2}+\left|Y_{\nu}\right|^{2}\left|H_{u}^{0} \tilde{\nu}^{c}\right|^{2} .
$$

The last term above is negligible in size compared to the other terms since it involves the Yukawa $Y_{\nu}$. Thus the coupling between this sector and the MSSM sector via the $H_{u}^{0}$ field is negligible. Now in the RG analysis there are no beta functions to turn $M_{\tilde{\nu}^{c}}^{2}$ negative and the quartic term is positive definite so the potential is bounded from below. Consequently the potential cannot support spontaneous breaking to generate a VEV for the field $\tilde{\nu}^{c}$ and thus $\left\langle\tilde{\nu}^{c}\right\rangle=0$. Further, the extrema equation for $\rho$ gives

$$
\langle\rho\rangle=-\frac{g_{\mathrm{BL}} M_{\mathrm{BL}}}{M_{\mathrm{BL}}^{2}+m_{\rho}^{2}}\left\langle\tilde{\nu}^{\dagger} \tilde{\nu}^{c}-\tilde{\nu}^{\dagger} \tilde{\nu}\right\rangle=0,
$$

and since $\langle\tilde{\nu}\rangle=0=\left\langle\tilde{\nu}^{c}\right\rangle$, one also has $\langle\rho\rangle=0$. Thus there is no spontaneous symmetry breaking in the system and the $B-L$ and consequently an R-parity is preserved. We add that the situation here is rather different from the Stueckelberg extensions introduced in [7-11] where $\rho$ receives a non-vanishing VEV. In [7-11], a non-vanishing VEV for $\rho$ would arise due the Stueckelberg sector mixing with the $\mathrm{U}(1)_{Y}$ sector of MSSM. In contrast in the minimal $B-L$ extension analyzed here there is no mixing with the $\mathrm{U}(1)_{Y}$ sector, and 
thus there is no VEV growth for $\rho$. Thus the entire mass growth in the $\mathrm{U}(1)_{B-L}$ sector occurs via the Stueckelberg mechanism. If we include a Fayet-Iliopoulos D term [80] then effectively the potential for $\tilde{\nu}^{c}$ is replaced by

$$
V_{\nu^{c}}=M_{\tilde{\nu}^{c}}^{2} \tilde{\nu}^{c \dagger} \tilde{\nu}^{c}+\frac{g_{\mathrm{BL}}^{2} m_{\rho}^{2}}{2\left(M_{\mathrm{BL}}^{2}+m_{\rho}^{2}\right)}\left(\tilde{\nu}^{c \dagger} \tilde{\nu}^{c}+\xi\right)^{2}+\left|Y_{\nu}\right|^{2}\left|H_{u}^{0} \tilde{\nu}^{c}\right|^{2}
$$

For the case when $\xi$ is negative a VEV growth for $\tilde{\nu}^{c}$ is possible and R-parity can be broken spontaneously. While an FI D-term naturally arises when the U(1) is anomalous the inclusion of an FI term for a non-anomalous U(1), which is the case we discuss, is superfluous, and we exclude it from the minimal model. Therefore, it is apparent that $R$ parity is always conserved within the minimal Stueckelberg $B-L$ extension of the MSSM. It is instructive to compare the above situation with the case where one may break B-L gauge symmetry by giving VEVs to fields which cary a B-L quantum number such that $3(B-L)$ is even. In this case $\mathrm{R}$ parity will be preserved while the $\mathrm{B}-\mathrm{L}$ gauge boson gets a mass. This possibility has been studied in many papers and more recently in [14]. However, as discussed in [14] since the right handed neutrinos are still present as they are needed for anomaly cancellation, the coupling between the right-handed neutrinos and the new Higgs fields can generate a tachyonic mass for the right handed sneutrino field in general which leads to R-parity violation in these models. This is in contrast to the mass growth for the Stueckelberg B-L model which provides a new framework where one can have R-parity conservation since one does not have new fields which can generate a negative mass term for the right-handed sneutrinos and the mass of the $B-L$ gauge boson is generated without the possibility of breaking $\mathrm{R}$ parity.

The analysis above follows with (minimal) universal boundary conditions on the soft scalar masses. However, since the nature of physics at the Planck scale is still largely unknown one should consider non-universalities as well. In this case one will have additional contribution to the mass squares of scalar masses [81, 82]. The analysis of [83-85] considers a contribution to $M_{\tilde{\nu}^{c}}^{2}$ arising from $\operatorname{Tr}\left(Q_{\mathrm{BL}} m^{2}\right)$ with

$$
S_{\mathrm{BL}} \equiv \operatorname{Tr}\left(Q_{\mathrm{BL}} m^{2}\right)=2\left(M_{\tilde{Q}}^{2}-M_{\tilde{L}}^{2}\right)+\left(M_{\tilde{e}^{c}}^{2}-M_{\tilde{d}^{c}}^{2}\right)+\left(M_{\tilde{\nu}^{c}}^{2}-M_{\tilde{u}^{c}}^{2}\right)
$$

under the constraint $\operatorname{Tr}\left(Y m^{2}\right)=0$, where

$$
S_{Y} \equiv \operatorname{Tr}\left(Y m^{2}\right)=M_{H_{2}}^{2}-M_{H_{1}}^{2}+\sum_{\text {gen }}\left(M_{\tilde{Q}^{2}}^{2}-2 M_{\tilde{u}^{c}}^{2}+M_{\tilde{d}^{c}}^{2}-M_{\tilde{L}^{2}}^{2}+M_{\tilde{e}^{c}}^{2}\right) .
$$

With the universal boundary conditions for only each family one has $S_{\mathrm{BL}}=0$. This can be achieved in minimal supergravity models where all scalars have the same soft mass term, or in $\mathrm{SO}(10)$ or $E_{6}$ scenarios where the boundary conditions tell us that all sfermions of one family should have the same soft mass term. However, with non-universal boundary conditions one will have in general $S_{\mathrm{BL}} \neq 0$. With inclusion of $S_{\mathrm{BL}}$ one could in principle turn $M_{\tilde{\nu}^{c}}^{2}$ negative. Such a situation is achieved with inclusion of specific constraints in the analysis of [83-85]. However, such constraints are not generic and the positivity $M_{\tilde{\nu}^{c}}^{2}$ may still be broadly valid even with inclusion of non-universalities of soft parameters. 
Now there are stringent bounds on an extra $B-L$ type gauge boson. One finds [86]

$$
M_{Z^{\prime}} / g_{\mathrm{BL}}>M_{\mathrm{BL}} \sim 6 \mathrm{TeV},
$$

which implies that for $g_{\mathrm{BL}} \sim 1$ the $B-L$ type $Z^{\prime}$ boson lies in the several TeV region. With a $Z^{\prime}$ of this mass scale, detection at LHC-7 may be difficult, both because of energy considerations and luminosity. Further, with the constraint as given by eq. (4.10) some of the other phenomenological implications of the model associated with the spin 0 and spin $\frac{1}{2}$ sectors will also be difficult to test. In what follows, we uncover a model which maintains the strict R-parity invariance of the minimal Stueckelberg $B-L$ extensions, even after mass growth of the $B-L$ gauge bosons, but with testable implications that are far more rich.

\section{$5 \quad \mathrm{U}(1)_{B-L} \otimes \mathrm{U}(1)_{X}$ Stueckelberg model}

As indicated in the last section, the $Z^{\prime}$ boson of the minimal $B-L$ model may be difficult to detect because of its heavy mass. We consider now an extension of the model of the previous section which overcomes this constraint and produces a $Z^{\prime}$ which is much lighter but still has $B-L$ interactions with matter. This extension includes a hidden sector $\mathrm{U}(1)_{X}$ which is anomaly free but allows for a mixing between the visible and the hidden sectors. The extended gauge group reads:

$$
\mathrm{SU}(3)_{C} \otimes \mathrm{SU}(2)_{L} \otimes \mathrm{U}(1)_{Y} \otimes \mathrm{U}(1)_{B-L} \otimes \mathrm{U}(1)_{X} .
$$

Thus we have Stueckelberg mass growth in the Abelian sector via the interaction

$$
\begin{aligned}
\mathcal{L}_{\mathrm{St}}=\int d^{2} \theta d^{2} \bar{\theta}\left[\left(M_{1} X\right.\right. & \left.+M_{2}^{\prime} C+S+\bar{S}\right)^{2} \\
& \left.+\left(M_{1}^{\prime} X+M_{2} C+S^{\prime}+\bar{S}^{\prime}\right)^{2}\right],
\end{aligned}
$$

where the model is invariant under the extended gauge transformations

$$
\begin{aligned}
\delta_{X}\left(X, S, S^{\prime}, C\right) & =\left(\epsilon_{X}+\bar{\epsilon}_{X},-M_{1} \epsilon_{X},-M_{1}^{\prime} \epsilon_{X}, 0\right) \\
\delta_{\mathrm{BL}}\left(C, S, S^{\prime}, X\right) & =\left(\epsilon_{\mathrm{BL}}+\bar{\epsilon}_{\mathrm{BL}},-M_{2}^{\prime} \epsilon_{\mathrm{BL}},-M_{2} \epsilon_{\mathrm{BL}}, 0\right)
\end{aligned}
$$

where $\epsilon_{X, B L}$ are infinitesimal chiral superfields. One can compute the mass matrix for the $\mathrm{U}(1)_{X}$ and the $\mathrm{U}(1)_{B-L}$ gauge vector bosons by going to the unitary gauge which in the basis $X_{\mu}, C_{\mu}$ gives

$$
M_{[\text {spin } 1]}^{2}=\left[\begin{array}{cc}
M_{1}^{2}+M_{1}^{\prime 2} & M_{1} M_{2}^{\prime}+M_{1}^{\prime} M_{2} \\
M_{1} M_{2}^{\prime}+M_{1}^{\prime} M_{2} & M_{2}^{2}+M_{2}^{\prime 2}
\end{array}\right] .
$$

Here $M_{1}^{\prime}, M_{2}^{\prime}$ are the mixing parameters and in the limit that $M_{1}^{\prime}, M_{2}^{\prime} \rightarrow 0$ we have that the masses of the $X_{\mu}, C_{\mu}$ bosons are $M_{1}, M_{2}$. The diagonalization gives us two massive vector bosons which we may call $Z^{\prime}, Z^{\prime \prime}$ where

$$
\begin{aligned}
X_{\mu} & =\cos \theta_{\mathrm{BL}} Z_{\mu}^{\prime}+\sin \theta_{\mathrm{BL}} Z_{\mu}^{\prime \prime}, \\
C_{\mu} & =-\sin \theta_{\mathrm{BL}} Z_{\mu}^{\prime}+\cos \theta_{\mathrm{BL}} Z_{\mu}^{\prime \prime} .
\end{aligned}
$$




\begin{tabular}{|ccc|}
\hline$f \bar{f}$ & $\Gamma\left(Z^{\prime} \rightarrow f \bar{f}\right) / \alpha_{\mathrm{BL}} M_{Z^{\prime}}$ & $\Gamma\left(Z^{\prime \prime} \rightarrow f \bar{f}\right) / \alpha_{\mathrm{BL}} M_{Z^{\prime \prime}}$ \\
\hline$\ell_{i}^{+} \ell_{i}^{-}$ & $\sin ^{2} \theta_{\mathrm{BL}} / 3$ & $\cos ^{2} \theta_{\mathrm{BL}} / 3$ \\
$\nu_{\ell_{i}} \nu_{\ell_{i}}$ & $\sin ^{2} \theta_{\mathrm{BL}} / 3$ & $\cos ^{2} \theta_{\mathrm{BL}} / 3$ \\
$q \bar{q}(q \neq t)$ & $f_{s} \sin ^{2} \theta_{\mathrm{BL}} / 9$ & $f_{s} \cos ^{2} \theta_{\mathrm{BL}} / 9$ \\
$t \bar{t}$ & $f_{s} f_{t, Z^{\prime}} \sin ^{2} \theta_{\mathrm{BL}} / 9$ & $f_{s} f_{t, Z^{\prime \prime}} \cos ^{2} \theta_{\mathrm{BL}} / 9$ \\
\hline
\end{tabular}

Table 1. The decay widths of the $Z^{\prime}$ and of the $Z^{\prime \prime}$ bosons into leptons and into quarks in the $\mathrm{U}(1)_{X} \otimes \mathrm{U}(1)_{B-L}$ Stueckelberg model where $\alpha_{\mathrm{BL}} \equiv g_{\mathrm{BL}}^{2} / 4 \pi$ and $f_{s}=\left(1+\frac{\alpha_{s}}{\pi}\right)$ and for $V=Z^{\prime}, Z^{\prime \prime}$, one has $f_{t, V}=\left(1+2 \frac{m_{t}^{2}}{M_{V}^{2}}\right)\left(1-\frac{4 m_{t}^{2}}{M_{V}^{2}}\right)$.

We consider now the case of small mixing, i.e., $M_{1}^{\prime}, M_{2}^{\prime} \ll M_{1}, M_{2}$ which implies $\tan \theta_{\mathrm{BL}} \ll$ 1. For small mixings the $Z^{\prime}$ boson lies mostly in the hidden sector with a small component proportional to $\tan \theta_{\mathrm{BL}}$ in the $B-L$ sector while the opposite holds for $Z^{\prime \prime}$. Here $Z^{\prime \prime}$ lies mostly in the $B-L$ sector with a small component proportional to $\tan \theta_{\mathrm{BL}}$ in the hidden sector.

Since $X_{\mu}$ lies in the hidden sector and has no couplings to the visible sector matter, the only couplings of $Z^{\prime}, Z^{\prime \prime}$ to the visible sector arises because of the couplings of $C_{\mu}$ to the visible sector matter. Using the couplings of $C_{\mu}$ one finds the couplings of $Z^{\prime}$ and $Z^{\prime \prime}$ to the fermions $\left(f_{i}\right)$ to be of the form

$$
\mathcal{L}_{Z^{\prime}, Z^{\prime \prime}}=\left(\bar{f}_{i} \gamma^{\mu} g_{\mathrm{BL}} Q_{\mathrm{BL}} f_{i}\right)\left[-\sin \theta_{\mathrm{BL}} Z_{\mu}^{\prime}+\cos \theta_{\mathrm{BL}} Z_{\mu}^{\prime \prime}\right]
$$

In the context of eq. (5.6) the constraint of eq. (4.10) gives two separate conditions, i.e.,

$$
\begin{aligned}
& M_{Z^{\prime}} / g_{\mathrm{BL}}>\sin \theta_{\mathrm{BL}} \times(6 \mathrm{TeV}), \\
& M_{Z^{\prime \prime}} / g_{\mathrm{BL}}>\cos \theta_{\mathrm{BL}} \times(6 \mathrm{TeV}) .
\end{aligned}
$$

It is clear that the constraint on the $Z^{\prime}$ is now considerably weakened relative to the constraint of eq. (4.10) if the mixing angle $\theta_{\mathrm{BL}}$ is small and one can have

$$
M_{Z^{\prime}} \ll 1 \mathrm{TeV}, \quad \text { Stueckelberg } \mathrm{U}(1)_{B-L} \otimes \mathrm{U}(1)_{X} .
$$

However, $Z^{\prime \prime}$ is still heavy since $\cos \theta_{\mathrm{BL}} \sim 1$ for small $\theta_{\mathrm{BL}}$.

In table 1 we give the decay widths of the $Z^{\prime}$ and $Z^{\prime \prime}$ bosons into leptons and into quarks. The relative strength of the $Z^{\prime}$ decay into quarks and leptons provides a distinctive signal for this model. Thus, for example, the ratio of the branching ratios of $Z^{\prime}$ into charged leptons vs into quarks (except into $t \bar{t}$ ) is given by $B R\left(Z^{\prime} \rightarrow \ell^{+} \ell^{-}\right) / B R\left(Z^{\prime} \rightarrow\right.$ $q \bar{q})=6 /\left(5\left(1+\alpha_{s} / \pi\right)\right)$. Further, in this model the decay width of the $Z^{\prime}$ and $Z^{\prime \prime}$ are related by

$$
\frac{\Gamma\left(Z^{\prime} \rightarrow \sum_{i} f_{i} \bar{f}_{i}\right)}{\Gamma\left(Z^{\prime \prime} \rightarrow \sum_{i} f_{i} \bar{f}_{i}\right)}=\tan ^{2} \theta_{\mathrm{BL}} \frac{M_{Z^{\prime}}}{M_{Z^{\prime \prime}}}
$$

eq. (5.9) implies that for the $Z^{\prime}$ mass in the sub TeV range, and the $Z^{\prime \prime}$ mass in the range above $6 \mathrm{TeV}$, and $\tan \theta_{\mathrm{BL}} \ll 1$ consistent with eq. (4.10), the ratio of the decay widths 

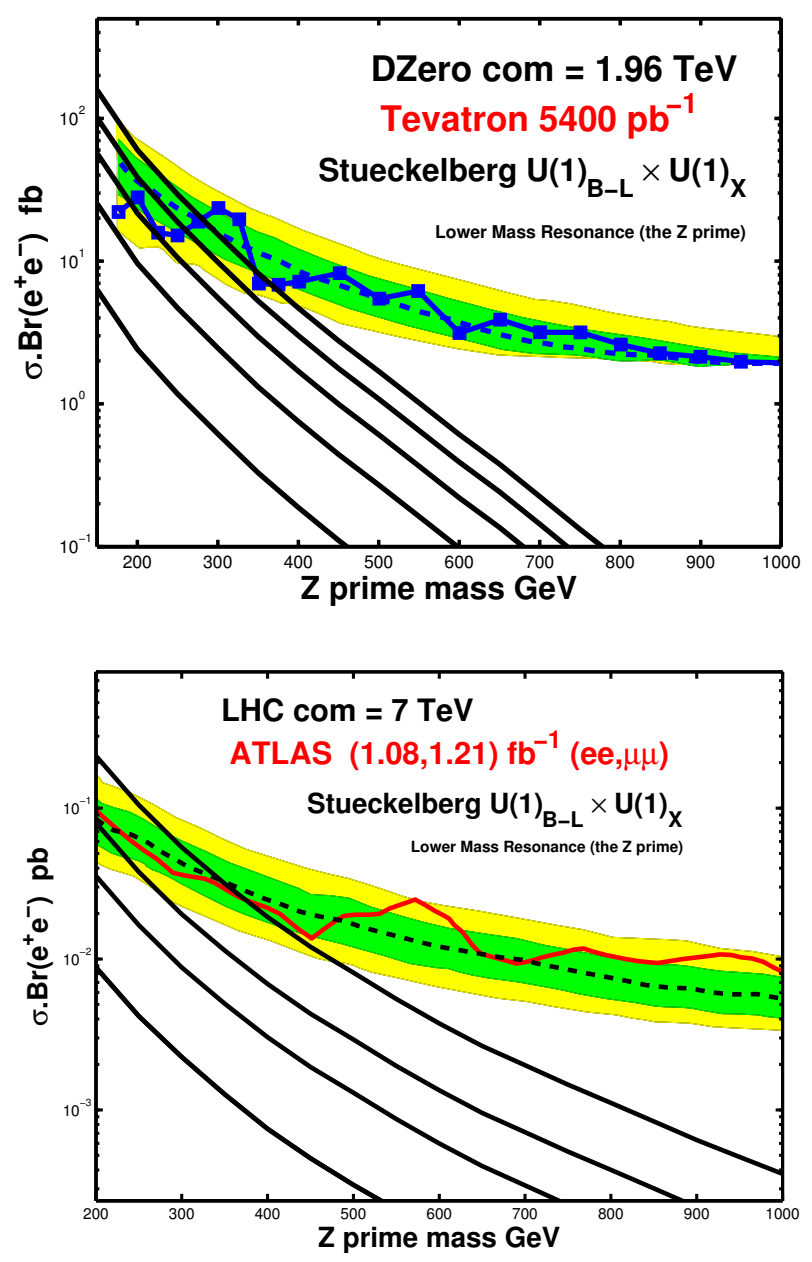

Figure 1. Upper panel: An exhibition of $\sigma\left(p p \rightarrow Z^{\prime}\right) \cdot \operatorname{Br}\left(Z^{\prime} \rightarrow e^{+} e^{-}\right)$vs the mass of the $Z^{\prime}$ resonance in the Stueckelberg $\mathrm{U}(1)_{B-L} \otimes \mathrm{U}(1)_{X}$ extension of MSSM at the Tevatron. Here $g_{\mathrm{BL}}=0.35$ and $\sin \theta_{\mathrm{BL}}$ takes on the values $0.01,0.05$ from the bottom to the top curves in the plot. The analysis assumes that the $Z^{\prime}$ decay into the hidden sector is suppressed. Lower panel: The same analysis at LHC-7 with $\sin \theta_{\mathrm{BL}}$ taking on the values $(0.01,0.02,0.03,0.05)$ from the bottom curve to the top in that order.

of $Z^{\prime}$ vs of $Z^{\prime \prime}$ can be vastly different, i.e., a decay width of $Z^{\prime}$ in the $\mathrm{MeV}$ range vs the decay width of $Z^{\prime \prime}$ in the hundreds of $\mathrm{GeV}$ range. Thus while the $Z^{\prime}$ will be a very narrow resonance, the $Z^{\prime \prime}$ will be a very broad resonance. The widths of the $Z^{\prime}$ and $Z^{\prime \prime}$ resonances is controlled by the mixing angle $\theta_{\mathrm{BL}}$. In our choice of the size of the mixing angle, we are guided by both the electroweak constraints, the constraint from the LHC, the constraint from the Tevatron, and the constraint from Dark Matter search limits. As a result of these constraints the size of the mixing angle is required to be small in order to satisfy all bounds as demonstrated in the analysis here.

It is also instructive to check the contribution of the new interactions to the muon anomalous moment which is now measured very accurately [87] so that the current error 


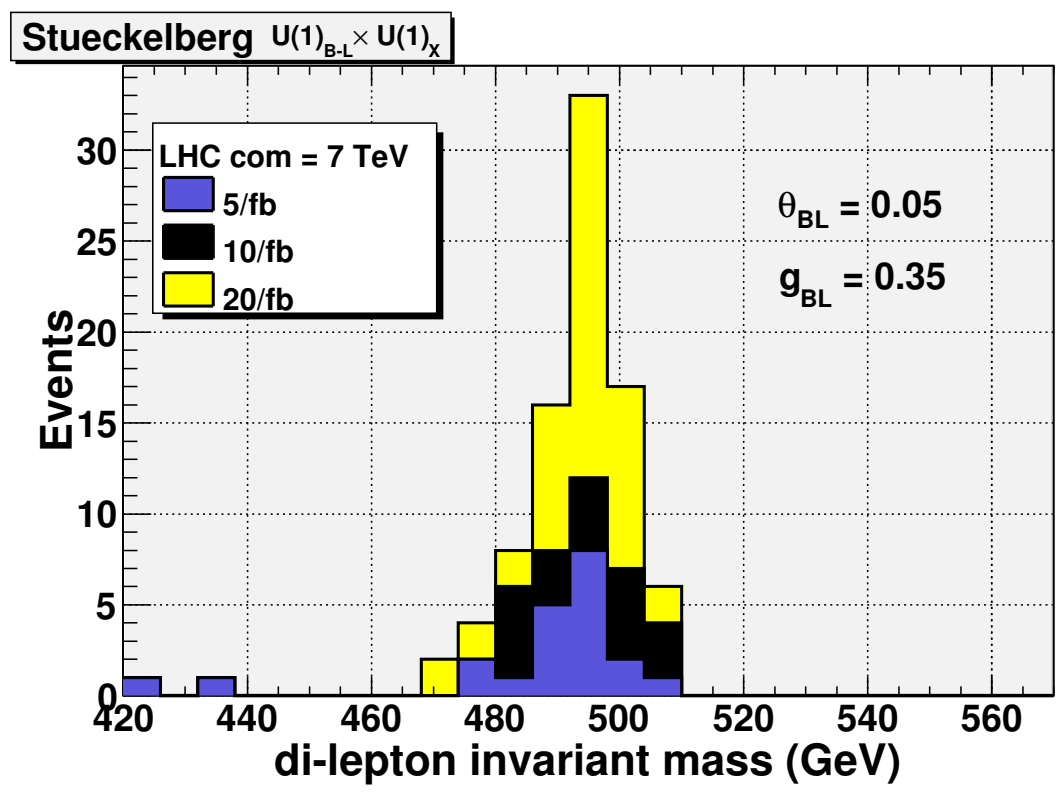

Figure 2. Exhibition of a $500 \mathrm{GeV} Z^{\prime}$ resonance in the Stueckelberg $\mathrm{U}(1)_{B-L} \otimes \mathrm{U}(1)_{X}$ model at LHC-7 with a variable luminosity from $5 \mathrm{fb}^{-1}$ to $20 \mathrm{fb}^{-1}$ with a $P_{T}$ cut on leptons of $P_{T}>30 \mathrm{GeV}$. Currently the LHC has analyzed $\sim 1 \mathrm{fb}^{-1}$ of luminosity. For a $Z^{\prime}$ resonance of $500 \mathrm{GeV}$ with $\theta_{\mathrm{BL}}=0.05$ and $g_{\mathrm{BL}} \sim g_{Y}$ the LHC would need about $5 \mathrm{fb}^{-1}$ to begin to see any $Z^{\prime}$ effect. With a very optimistic $20 \mathrm{fb}^{-1}$, the $Z^{\prime}$ signal will be strong and $Z^{\prime}$ should be visible with the mixings and masses of the size discussed.

in the determination is given by $\Delta\left(g_{\mu}-2\right)=1.2 \times 10^{-9}$. The contribution of the $Z^{\prime}$ and of the $Z^{\prime \prime}$ bosons to the anomalous moment is given by

$$
\Delta\left(g_{\mu}-2\right)=\frac{g_{\mathrm{BL}}^{2} m_{\mu}^{2}}{24 \pi^{2}}\left[\frac{\sin ^{2} \theta_{\mathrm{BL}}}{M_{Z^{\prime}}^{2}}+\frac{\cos ^{2} \theta_{\mathrm{BL}}}{M_{Z^{\prime \prime}}^{2}}\right]
$$

Using the LEP constraint of eq. (5.7) one finds that the contributions of the new interactions is

$$
\Delta\left(g_{\mu}-2\right) \leq \frac{m_{\mu}^{2}}{12 \pi^{2} M_{\mathrm{BL}}^{2}}
$$

and a substitution of $M_{\mathrm{BL}} \sim 6 \mathrm{TeV}$ gives a rather small contribution, i.e., $\Delta\left(g_{\mu}-2\right) \leq$ $O(1) \times 10^{-12}$. Remarkably in this case the LEP constraint of eq. (5.7) is stronger than the constraint arising from the very precise measurement of $g_{\mu}-2$.

\subsection{Production of vector resonances}

The fact that the $Z^{\prime}$ boson could have a low mass has important phenomenological implications. From table 1 we note that the decay width of the $Z^{\prime}$ boson is proportional to $\sin ^{2} \theta_{\mathrm{BL}}$ and $\operatorname{since} \sin \theta_{\mathrm{BL}}$ is small, the decay width is relatively small, i.e., with the mass of the $Z^{\prime}$ in the sub $\mathrm{TeV}$ region, its decay width would be in the $\mathrm{MeV}$ range and thus the Stueckelberg $Z^{\prime}$ is a very narrow resonance. A narrow resonance of this type should be 
testable in collider experiments much like the hypercharge Stueckelberg $Z^{\prime}$ on which the DØ currently has experimental bounds [88]. Further, the decay of the Stueckelberg $Z^{\prime}$ into leptonic channels will be much more than in the hadronic channels because the branching ratios are proportional to $(B-L)^{2}$. Thus one can discriminate a $B-L$ Stueckelberg $Z^{\prime}$ boson by a study of its branching ratios. Such a resonance could be produced in the Drell-Yan process at the LHC and the Tevatron via

$$
p p(p \bar{p}) \rightarrow Z^{\prime} \rightarrow \ell \bar{\ell}, q \bar{q}
$$

In figure 1 we show the predictions for the $Z^{\prime}$ cross section times the branching ratio into $e^{+} e^{-}$in the $\mathrm{U}(1)_{B-L} \otimes \mathrm{U}(1)_{X}$ extension of the Standard Model. Cross sections and event rates are calculated by implementing the couplings into PYTHIA and PGS [89, 90]. The bottom panel shows the limits on the production cross section for $\sigma\left(p p \rightarrow Z^{\prime} \rightarrow e \bar{e}\right)$ at $\sqrt{s}=7 \mathrm{TeV}$ with the recently released $\sim 1 \mathrm{fb}^{-1}$ run [91, 92]. For these curves we take $g_{\mathrm{BL}}$ to be have the same value as the hypercharge gauge coupling $g_{Y}$ and we let $\sin \theta_{\mathrm{BL}}$ run from 0.01 to 0.05 . The cross section for other values of the product $g_{\mathrm{BL}} \sin \theta_{\mathrm{BL}}$ can be estimated by the scaling in the cross section which at the $Z^{\prime}$ resonance scales like $g_{\mathrm{BL}}^{2} \sin ^{2} \theta_{\mathrm{BL}}$. The top panel gives a similar analysis for the Tevatron using the $\mathrm{D} \varnothing$ data with $5.4 / \mathrm{fb}$ of integrated luminosity [88]. From the analysis of figure 1 we observe that at present the Tevatron bound is about as strong as the present LHC bound. However, the LHC will surpass the Tevatron very soon. Indeed, the $Z^{\prime}$ produced in the model can exist with a much lower mass $[10,11,93,94]$ than the $Z^{\prime}$ models presently excluded by ATLAS [91, 92] and CMS [95]. In figure 2 we display the number of events as a function of the di-lepton invariant mass. Here one finds that with an optimistic choice of an integrated luminosity of $20 \mathrm{fb}^{-1}$ the number of dileptonic events in excess of 30 in the peak mass bin and should be visible. Thus a $Z^{\prime}$ mass of $500 \mathrm{GeV}$ with a mixing angle $\theta_{\mathrm{BL}}=0.05$ and $g_{\mathrm{BL}}=g_{Y}$ is a promising candidate for discovery. We note that in addition to the dilepton channel, other channels such as di-jet or fully hadronic channels are also possible discovery channels. However, these channels have much larger backgrounds. This is the reason why ATLAS and CMS have dedicated studies for narrow Z prime resonances focusing on the dilepton channels while the hadronic channels do not provide a very strong constrain in this case. Thus the cleanest channel to discover the signal of a narrow resonance that decays into leptons with a significant branching ratio is the Drell-Yan dilepton channel as the backgrounds fall dramatically beyond the $\mathrm{Z}$ boson mass.

\subsection{Production and decay of the scalars $\rho$ and $\rho^{\prime}$}

In addition to the $Z^{\prime}$ phenomenology there are other sectors where new phenomena can arise. One of these relates to the scalar components $\rho_{X}$ and $\rho_{\mathrm{BL}}$ of $S+\bar{S}$ and of $S^{\prime}+\bar{S}^{\prime}$ that remain in the bosonic sector after $Z^{\prime}$ and $Z^{\prime \prime}$ gain mass by the Stueckelberg mechanism. These fields mix with the D-terms so that one has the following set in the Lagrangian

$$
\rho_{\mathrm{BL}}\left(M_{1} D_{\mathrm{BL}}+M_{2}^{\prime} D_{X}\right)+\rho_{X}\left(M_{1}^{\prime} D_{\mathrm{BL}}+M_{2} D_{X}\right)
$$


Elimination of the D-terms gives the following mass matrix in the $\rho_{X}$ and $\rho_{\mathrm{BL}}$ basis

$$
M_{\text {[spin 0] }}^{2}=\left[\begin{array}{cc}
M_{1}^{2}+M_{2}^{\prime 2}+m_{X}^{2} & M_{1} M_{1}^{\prime}+M_{2} M_{2}^{\prime} \\
M_{1} M_{1}^{\prime}+M_{2} M_{2}^{\prime} & M_{1}^{\prime 2}+M_{2}^{2}+m_{\mathrm{BL}}^{2}
\end{array}\right],
$$

where we have also included the soft contributions to masses for $\rho_{X}$ and $\rho_{\mathrm{BL}}$. We note that the structure of the spin zero mass squared matrix given by eq. (5.14) is different compared to the mass ${ }^{2}$ matrix given by eq. (5.4). The reason for this is that while the vector mass squared matrix arises directly from the Stueckelberg term eq. (5.2), the mass squared matrix of eq. (5.14) arises from the mixing given by eq. (5.13). The mass matrix of eq. (5.14) gives two mass eigenstates $\rho$ and $\rho^{\prime}$ with eigenvalues $M_{\rho}$ and $M_{\rho^{\prime}}$. The mass parameters $M_{1}^{\prime}, M_{2}^{\prime}$ can define the mixing and when the mixing is small, $M_{\rho}^{2} \rightarrow M_{1}^{2}+m_{X}^{2}$ and $M_{\rho^{\prime}}^{2} \rightarrow M_{2}^{2}+m_{\mathrm{BL}}^{2}$. With $M_{\rho}$ in the sub $\mathrm{TeV}$ range $M_{\rho^{\prime}}$ may have a mass in the several $\mathrm{TeV}$ range. These mass eigenstates are admixtures of $\rho_{X}$ and $\rho_{\mathrm{BL}}$ so that $\rho_{X}=\cos \theta_{\mathrm{BL}}^{\prime} \rho+\sin \theta_{\mathrm{BL}}^{\prime} \rho^{\prime}$ and $\rho_{\mathrm{BL}}=-\sin \theta_{\mathrm{BL}}^{\prime} \rho+\cos \theta_{\mathrm{BL}}^{\prime} \rho^{\prime}$. For the case when the soft terms are absent, the eigenvalues of the mass squared matrix of eq. (5.14) is are identical despite the very different looks of the matrices of eq. (5.4) and eq. (5.14). This can be seen by the following unitary transformation

$$
U^{\dagger} M_{[\operatorname{spin} 1]}^{2} U=M_{[\text {spin } 0]}^{2},
$$

where the unitary matrix that connects the spin 1 and spin 0 matrix is given by

$$
U=\left(\begin{array}{cc}
\cos \xi & \sin \xi \\
-\sin \xi & \cos \xi
\end{array}\right), \quad \tan \xi=\frac{M_{1}^{\prime}-M_{2}^{\prime}}{M_{1}+M_{2}} .
$$

This result shows that the eigenvalues for the matrices $M_{\text {[spin 1] }}^{2}$ and $M_{\text {[spin 0] }}^{2}$ are the same in the limit of vanishing soft masses for the scalars. Now it is assumed that all the matter fields in the visible sector do not carry any $\mathrm{U}(1)_{X}$ quantum numbers, i.e., $Q_{X}=0$ for quarks, leptons and the Higgs fields. Further, following the analysis of section 6 , it is straightforward to establish that the quartic term $\left(\tilde{\nu}^{c \dagger} \tilde{\nu}^{c}\right)^{2}$ has a positive co-efficient in the scalar potential. Thus once again since there are no couplings in the model to turn $M_{\tilde{\nu}^{c}}^{2}$ negative, there is no spontaneous violation of R-parity also in this extended model while the $B-L$ gauge boson develops a mass via the Stueckelberg mechanism.

From the discussion preceding eq. (5.15), it is clear that the field $\rho_{X}$ has no coupling with the visible sector while $\rho_{\mathrm{BL}}$ has couplings of the form $g_{\mathrm{BL}} M \rho_{\mathrm{BL}} \tilde{\tilde{f}}_{i} Q_{\mathrm{BL}} \tilde{f}_{i}$. One then has the following interactions of $\rho$ and $\rho^{\prime}$ with sfermions

$$
\mathcal{L}_{\rho \tilde{f}^{\dagger} \tilde{f}}=-\sin \theta_{\mathrm{BL}}^{\prime} g_{\mathrm{BL}} M_{1} \tilde{f}_{i}^{\dagger} Q_{\mathrm{BL}} \tilde{f}_{i} \rho+\cos \theta_{\mathrm{BL}}^{\prime} g_{\mathrm{BL}} M_{1} \tilde{f}_{i}^{\dagger} Q_{\mathrm{BL}} \tilde{f}_{i} \rho^{\prime} .
$$

Eq. (5.17) allows the decay of the $\rho\left(\rho^{\prime}\right)$ via its couplings to the sfermions. If kinematically allowed $\rho\left(\rho^{\prime}\right)$ will decay into leptons $+E_{T}^{\text {miss }}$ or into jets $+E_{T}^{\text {miss }}$ where $E_{T}^{\text {miss }}$ contains at least two neutralinos $\chi^{0}$ (here $\chi^{0}$ is the lightest neutralino (LSP) of the $\mathrm{U}(1)_{B-L} \otimes \mathrm{U}(1)_{X}$ combined sector - see section 6$)$. However, an interesting situation arises when the mass of $\rho\left(\rho^{\prime}\right)$ is smaller than $2 M_{\chi^{0}}$. In this case $\rho\left(\rho^{\prime}\right)$ cannot decay into the final states with 2 

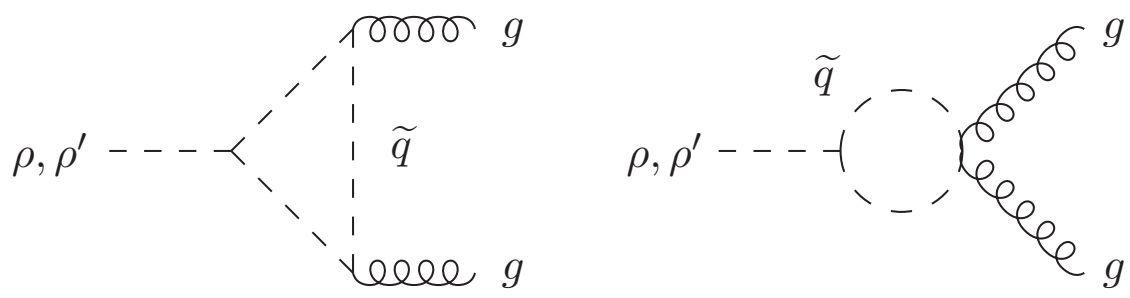

Figure 3. Diagrams giving rise to the production of the Stueckelberg scalars, $\rho, \rho^{\prime}$ at the lowest order.

LSPs and only the decays into the Standard Model particles are allowed. Such decays can occur via loops and the final states will consist of $g g, f_{i} \bar{f}_{i}, W W, Z Z, \gamma Z, \gamma \gamma$. There are many diagrams that contribute. The dominant one relevant to the model we study here with real scalars $\rho$ and $\rho^{\prime}$ are the gluon fusion diagrams (see figure 3 ).

From eq. (5.17) the interactions of $\rho$ and $\rho^{\prime}$ to the mass diagonal squarks are given by the following interaction

$$
\begin{aligned}
\mathcal{L}_{\left(\rho, \rho^{\prime}\right) \tilde{q}^{\dagger} \tilde{q}}= & -g_{\rho} M_{1} \cos \left(2 \theta_{\tilde{q}_{i}}\right)\left(\tilde{q}_{1 i}^{\dagger} \tilde{q}_{1 i} \rho-\tilde{q}_{2 i}^{\dagger} \tilde{q}_{2 i} \rho\right)-g_{\rho} M_{1} \sin \left(2 \theta_{\tilde{q}_{i}}\right)\left(\tilde{q}_{1 i}^{\dagger} \tilde{q}_{2 i} \rho+\tilde{q}_{2 i}^{\dagger} \tilde{q}_{1 i} \rho\right) \\
& +\left(\rho \rightarrow \rho^{\prime},-\sin \theta_{\mathrm{BL}} \rightarrow \cos \theta_{\mathrm{BL}}\right) .
\end{aligned}
$$

with the $B-L$ dependance encoded via

$$
g_{\rho}=\frac{1}{3} g_{\mathrm{BL}} \sin \theta_{\mathrm{BL}}^{\prime}
$$

and where $i$ runs over the squark flavors. Now while the $\rho, \rho^{\prime}$ vertices allow couplings with squark mass eigenstates, where the two states couple to are either the same state or different states, the gluino only couples to squark states, where both states have the same mass. Thus in eq. (5.18) only the interaction terms proportional to $\cos 2 \theta_{\tilde{q} i}$ enter in the gluon fusion diagram. As such, the decay width of the $\rho$ to gluons is given by

$$
\Gamma(\rho \rightarrow g g)=\frac{g_{\rho}^{2} \alpha_{s}^{2} M_{\rho}^{3} M_{1}^{2}}{512 \pi^{3}}\left|\sum_{a=1,2 ; i}(-1)^{1+a} \cos \left(2 \theta_{\tilde{q}_{i}}\right) \frac{L_{1}\left(r_{a i}\right)}{m_{\tilde{q}_{a i}}^{2}}\right|^{2}
$$

with $r_{a i}=M_{\rho}^{2} /\left(4 m_{\tilde{q}_{a i}}^{2}\right)$, and $L_{1}(r)$ is a loop function defined by [96]

$$
L_{1}(r)=r^{-2}[r-f(r)], \quad f(r)= \begin{cases}\arcsin ^{2}(\sqrt{r}) & r \leq 1 \\ -\frac{1}{4}\left(\log \frac{1+\sqrt{1-r^{-1}}}{1-\sqrt{1-r^{-1}}}-i \pi\right)^{2} & r>1 .\end{cases}
$$

As a consequence of the symmetry of gauge interactions one also has

$$
\Gamma\left(\rho^{\prime} \rightarrow g g\right)=\frac{M_{\rho^{\prime}}^{3}}{M_{\rho}^{3}} \cot ^{2} \theta_{\mathrm{BL}}^{\prime} \Gamma(\rho \rightarrow g g) .
$$




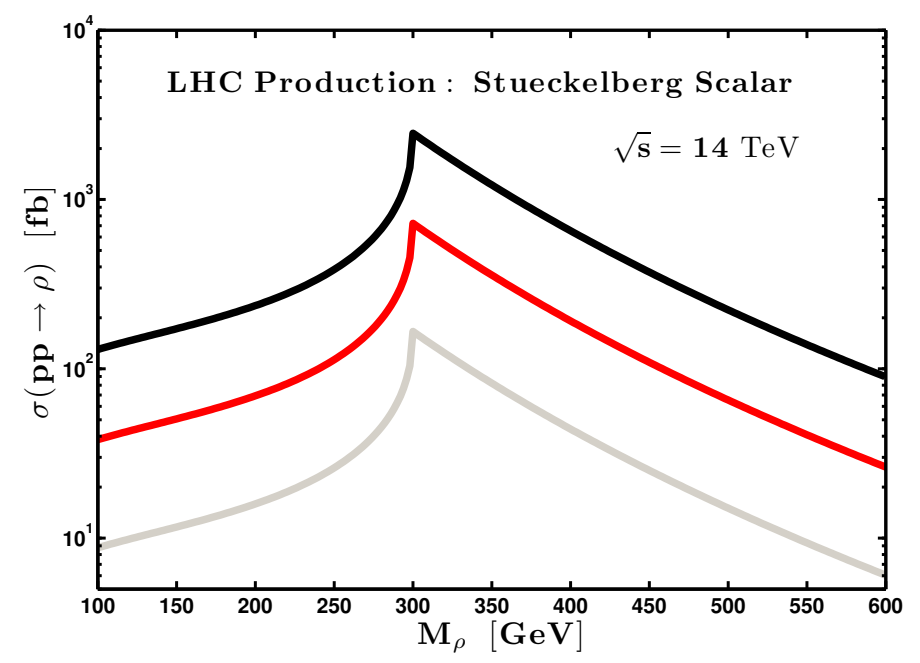

Figure 4. A display of the production cross section $\sigma(p p \rightarrow \rho)$ from gluon fusion as a function of the mass of the Stueckelberg scalar $\rho$ at the LHC with $\sqrt{s}=14 \mathrm{TeV}$ for several combinations of $\theta_{\mathrm{BL}}^{\prime}$ and $g_{\mathrm{BL}}$ for the case which maximizes the production for the MSSM sector, $\left|\cos 2 \theta_{\tilde{t}}\right|=1$, i.e. $\left|A_{t}\right| \tan \beta=$ $|\mu|$. From bottom to top the curves have $\left(\theta_{\mathrm{BL}}^{\prime}, g_{\mathrm{BL}}\right)=(\pi / 6,0.65),(\pi / 2,0.65),(\pi / 2,1.2)$, where the top curve is close to the theoretical upper limit on the production. The kink appears at the point where $M_{\rho} /\left(2 m_{\tilde{t}_{1}}\right)=1$ and the analysis has the other squarks and the gluino much heavier than the lighter stop.

Further the partonic production cross section of $\rho$ is given by

$$
\hat{\sigma}(g g \rightarrow \rho)=\frac{g_{\rho}^{2} \alpha_{s}^{2} M_{1}^{2}}{256 \pi M_{\rho}^{4}}\left|\sum_{a=1,2 ; i}(-1)^{1+a} \cos \left(2 \theta_{\tilde{q}_{i}}\right) r_{a i} L_{1}\left(r_{a i}\right)\right|^{2} \delta\left(1-M_{\rho}^{2} / \hat{s}\right) .
$$

The hadronic production cross section relevant to the search for $\rho$ at the LHC is $\sigma(p p \rightarrow \rho)$ and is given by a convolution with the parton distribution functions for the gluon, which at leading order in the narrow width approximation is given by

$$
\sigma(p p \rightarrow \rho)(s)=\tau_{\rho} \frac{d L_{g g}^{p p}}{d \tau_{\rho}} \hat{\sigma}(g g \rightarrow \rho)
$$

Here $\sqrt{s}$ is the $p p$ center-of-mass energy, $\tau_{\rho}=M_{\rho}^{2} / s$, and $d L_{g g}^{p p} / d \tau$ is given by

$$
\frac{d L_{g g}^{p p}}{d \tau}=\int_{\tau}^{1} \frac{d x}{x} f_{g / p}(x, Q) f_{g / p}\left(\frac{\tau}{x}, Q\right)
$$

where $f_{g / p}$ is the parton distribution function for finding the gluon inside a proton with momentum fraction $x$ at a factorization scale $Q$. A numerical analysis shows that $\sigma(p p \rightarrow$ $\rho$ ) can lie in the range $\mathrm{O}(1000) \mathrm{fb}$ in the most optimal part of the parameter space for producing the $\rho$.

The final decay modes of the $\rho$ can produce visible signatures at the LHC, and branching ratios will generally be different from the Standard Model Higgs $h_{\mathrm{SM}}$. Thus $h_{\mathrm{SM}}$ has 
both tree level decays into the final states $b \bar{b}, \tau \bar{\tau}, c \bar{c}$ as well as decays via loop diagrams into $g g, W W, Z Z, Z \gamma, \gamma \gamma$. For a Higgs boson mass of $100 \mathrm{GeV}$, dominant decays modes are the tree level decay modes with $b \bar{b}$ decay being almost $80 \%$. Among the loop decays the dominant decay is $g g$ and sub-dominant decays are $W W$ (off shell) and $\gamma \gamma$ at a Higgs mass of $100 \mathrm{GeV}$. Now suppose the tree decays of the Higgs were suppressed, then the decay of the Higgs to $\gamma \gamma$ will have a branching ratio of $\sim 2.5 \times 10^{-2}$. The decay of the $\rho$ parallels this case since there are no tree decays of the $\rho$. In the analysis below we will use the above branching ratio to get an approximate estimate of $\gamma \gamma$ event for the $\rho$ decay. An analysis of $p p \rightarrow \rho$ at the LHC at $\sqrt{s}=14 \mathrm{TeV}$ is given in figure 4 . One finds that the cross section at $M_{\rho}=100 \mathrm{GeV}$ for the maximal case with $\left(\theta_{\mathrm{BL}}^{\prime}, g_{\mathrm{BL}}\right)=(\pi / 2,1.2)$ is $\sim 100 \mathrm{fb}$. At $200 \mathrm{fb}^{-1}$ of integrated luminosity at the LHC at $\sqrt{s}=14 \mathrm{TeV}$, one will have $2 \times 10^{5} \rho$ events when $M_{\rho}=100 \mathrm{GeV}$. Using $B R(\rho \rightarrow \gamma \gamma)=2.5 \times 10^{-2}$ one finds $\sim 5000 \gamma \gamma$ events before kinematic and efficiency cuts. We note that the photons coming from the $\gamma \gamma$ signal will be monochromatic carrying roughly half the mass of the decaying particle. Thus the $\gamma \gamma$ signal arising from the decay of the $\rho$ would be distinguishable from the $\gamma \gamma$ signal from the Higgs decay if the masses of the two are significantly separated. A $\rho$ mass of $100 \mathrm{GeV}$ would imply a $Z^{\prime}$ mass of also $100 \mathrm{GeV}$ assuming no soft terms in the $\rho$ sector. A $Z^{\prime}$ mass of $100 \mathrm{GeV}$ is consistent with the current data if either the mixing angle $\theta_{\mathrm{BL}}$ is small or the $Z^{\prime}$ decays dominantly into the hidden sector (see section 6.2). We note also that while the mass of the $Z^{\prime}$ and the mass of $\rho$ are the same in the absence of soft breaking terms for $\rho$, the couplings of the $Z^{\prime}$ to fermions and of $\rho$ to squarks can be of very different sizes. This is apparent from eq. (5.16). Hence the possibility arises of being able to discover both the $\rho$ and the $Z^{\prime}$. However it is also quite possible that only one resonance may be visible depending on the overall size of the Stueckelberg masses and the individual couplings of the two states.

The production cross section for $p p \rightarrow \rho, \rho^{\prime}$ bears resemblance to the analysis of [17] and is closely related to canonical Higgs production (see e.g. [96, 97]) but is restricted by the form of the couplings as given in the $B-L$ Stueckelberg extension. We add that recently several models with scalars have been studied in the literature which can produce large production enhancements relative to the SM higgs production (see e.g [98-101]). The production of $\rho$ does not receive enhancements of the size studied above, but nevertheless does produce event rates that can be measured at the LHC-14 with larger luminosity as was detailed above.

We note that very recently the LHC has put new constraints on the allowed mass of the Standard Model Higgs Boson $h_{\mathrm{SM}}$. Preliminary analyses based on those reported at EPS 2011 and at Lepton-Photon 2011 [102, 103] imply that the SM Higgs boson has a mass below $\sim 145 \mathrm{GeV}$. The above result is compatible with the SUGRA models which typically indicate a Higgs mass below $\sim 140 \mathrm{GeV}$. Because the production of $\rho$ relative to the $h_{\mathrm{SM}}$ differs markedly via their couplings, as discussed above, the production of the two fields could be distinguished with sufficient luminosity. This is possible if the $h_{\mathrm{SM}}$ resonance and the $\rho$ resonance are sufficiently separated in mass. In addition, because the production of $\rho$ is weaker than $h_{\mathrm{SM}}$, the golden channels such as $Z Z, W W$ remain available where $h_{\mathrm{SM}}$ has been ruled out to have such a mass. Searches for $M_{\rho} \sim(200-500) \mathrm{GeV}$ will however have to wait for upgraded luminosity at the LHC. 


\section{Neutral Dirac and Majorana components of dark matter}

\subsection{Majorana dark matter}

The $\mathrm{U}(1)_{B-L} \otimes \mathrm{U}(1)_{X}$ Stueckelberg extension of MSSM have new implications for the nature of dark matter. Specifically in the neutralino sector we have in addition to the MSSM neutralinos, extra gauginos and stinos, where the stino is the analogue of the higgsino. Thus from the gauge supermultiplets $X=\left(X_{\mu}, \lambda_{X}, D_{X}\right)$ and $C=\left(C_{\mu}, \lambda_{C}, D_{C}\right)$ we can construct two gaugino states which we label as $\Lambda_{X}, \Lambda_{\mathrm{BL}}$. Similarly from the chiral multiplets $S+\bar{S}$ and $S^{\prime}+\bar{S}^{\prime}$ we can construct two higgsino states $\psi_{S}, \psi_{S^{\prime}}$. These four neutralino states in the Stueckelberg sector have no mixings with the MSSM neutralinos. Thus the neutralino mass matrix in the $\mathrm{U}(1)_{X} \otimes \mathrm{U}(1)_{B-L}$ extension of MSSM has the form

$$
\mathcal{M}_{\text {neutralino }}=\left(\begin{array}{c|c}
\mathcal{M}_{s t} & 0_{4 \times 4} \\
\hline 0_{4 \times 4} & \mathcal{M}_{\mathrm{MSSM}}
\end{array}\right)
$$

Specifically the neutralino mass terms in the $\mathrm{U}(1)_{X} \otimes \mathrm{U}(1)_{B-L}$ sector are given by

$$
\begin{aligned}
\mathcal{L}^{\text {mass }} & =-Z^{T} \mathcal{M}_{s t} Z, \\
Z^{T} & =\left(\psi_{S}, \psi_{S^{\prime}}, \Lambda_{B-L}, \Lambda_{X}\right),
\end{aligned}
$$

where the $4 \times 4$ sub-block of the $\mathrm{U}(1)_{B-L} \otimes \mathrm{U}(1)_{X}$ sector has the form (omitting for simplicity the soft terms)

$$
\mathcal{M}_{s t}=\left(\begin{array}{cc}
0_{2 \times 2} & m \\
m^{T} & 0_{2 \times 2}
\end{array}\right)_{4 \times 4}, m=\left(\begin{array}{ll}
M_{1} & M_{2}^{\prime} \\
M_{1}^{\prime} & M_{2}
\end{array}\right)_{2 \times 2} .
$$

We can diagonalize the neutralino mass matrix in the $\mathrm{U}(1)_{X} \otimes \mathrm{U}(1)_{B-L}$ sector by an orthogonal transformation $Z=O X$ so that

$$
X^{T} O^{T} \mathcal{M}_{s t} O X=\operatorname{diag}\left(m_{\chi_{5}^{0}}, m_{\chi_{6}^{0}}, m_{\chi_{7}^{0}}, m_{\chi_{8}^{0}}\right) .
$$

Now the generalization of the matter Lagrangian reads

$$
\mathcal{L}_{\text {matter }}=\left.\bar{\Phi}_{m} e^{2 g_{\mathrm{BL}} Q_{\mathrm{BL}} C+2 g_{X} Q_{X} X} \Phi_{m}\right|_{\theta^{2} \bar{\theta}^{2}}
$$

and gives a coupling of the type $\bar{\Lambda}_{B-L} f_{L} \tilde{f}_{L}^{*}$, where $\left(f_{L}, \tilde{f}_{L}\right)$ are a chiral fermion and a chiral scalar, which leads to couplings of the Stueckelberg sector neutralinos with matter of the form $\bar{\chi}_{k}^{0} f_{L} \tilde{f}_{L}^{*}(k=5-8)$. Thus we note that even though the neutralino mass matrix does not have a mixing between the MSSM and the Stueckelberg sectors, the neutralino in the Stueckelberg sector can decay into the least massive supersymmetric particle (LSP) which may lie in the MSSM sector. The way it occurs is as follows: The neutralinos $\chi_{k}^{0}(k=5-8)$ have fermion-sfermion interactions as indicated above, while the neutralinos in the MSSM also have similar type interactions. If the mass $m_{\chi_{k}^{0}}>m_{\chi_{1}^{0}}$ we will have decays of the type $\chi_{k}^{0} \rightarrow \overline{\tilde{f}}_{i} f_{i} \rightarrow \bar{f}_{i} f_{i} \chi_{1}^{0}+\cdots$. Thus there is only one stable Majorana supersymmetric particle in the combined MSSM and Stueckelberg system. On the other hand if, for example, $\chi_{5}^{0}$ is the lightest neutralino then the LSP will lie in the Stueckelberg sector. In this case 
the $\chi_{\mathrm{st}}^{0}=\chi_{5}^{0}$ would be a dark matter candidate (the notation st denotes stueckelberg and does not imply preference of the stino component over the gaugino component). Its properties are expected to be similar to those of the bino LSP of the MSSM. For the case of a thermal relic, the annihilation of $\chi_{\text {st }}$ will occur via the t-channel squark exchange so that (dropping the superscript 0 from here on) $\chi_{\mathrm{st}}+\chi_{\mathrm{st}} \rightarrow f_{i} \bar{f}_{i}$, as wells $\chi_{\mathrm{st}}+\tilde{f}_{\mathrm{MSSM}} \rightarrow \mathrm{SM} \mathrm{SM}^{\prime}$, $\chi_{\mathrm{st}}+\chi_{\mathrm{MSSM}} \rightarrow \mathrm{SM} \mathrm{SM}^{\prime}$, where the last two cases indicate that the the coannihilations will generally occur [32, 48-50, 73, 74] (for a review see [104]). For the direct annihilations, unlike the annihilation of MSSM neutralinos, there are no direct channel $Z$ or Higgs pole exchange diagrams and consequently final states such as $W W, Z Z, Z H, H H$ are absent at the tree level. For the case of co-annihilations this is modified. If $\rho$ is of low mass, as discussed in the previous section, the stop should be relatively light to accommodate a signal of $\rho$. In this case the relic density can be satisfied via stop co-annihilations $[120,121]$.

Next, we discuss the the direct detection of $\chi_{\mathrm{st}}$. Specifically there are no $t$-channel Higgs or $Z$ pole exchange contributions to the direct detection rates for this case at the tree level. As pointed out in ref. [105-107] it is important to include contributions arising in the spin independent scattering cross section from the twist-2 operators

$$
f_{p} / m_{p} \ni \sum_{q=u, d, s} f_{q} f_{T q}+\sum_{q=u, d, s, c, b} \frac{3}{4}(q(2)+\bar{q}(2)) g_{q}^{(1)}+\ldots
$$

where the additional terms are suppressed and $q(2), \bar{q}(2)$ are matrix elements and are given in [107]. Specifically $g_{q}^{(1)}$ is given by, in the limit of massless quarks

$$
g_{q}^{(1)} \simeq \frac{M_{\chi_{\mathrm{st}}}}{\left(m_{\tilde{q}}^{2}-M_{\chi_{\mathrm{st}}}^{2}\right)^{2}} \frac{a_{q}^{2}+b_{q}^{2}}{2}
$$

where $a_{q}^{2}+b_{q}^{2}=g_{\mathrm{BL}}^{2} Q_{\mathrm{BL}}^{2}=g_{\mathrm{BL}}^{2} / 9$. In addition, there are terms of size $\sum_{q=u, d, s} f_{q} f_{T q}$ (where $f_{q}, f_{T q}$ are given in $[105,106,108,109]$ ). Here terms in $f_{q}$ that are proportional to $a_{q}^{2}+b_{q}^{2}$ are suppressed by a factor of 4 relative to $g_{q}^{(1)}$ [107]. Terms in $f_{q}$ also contain $a_{q}^{2}-b_{q}^{2} \propto g_{\mathrm{BL}}^{2} Q_{\mathrm{BL}}^{2} \sin 2 \theta_{\tilde{q}}$ and are ultra suppressed by the smallness of the squark mixing angle. For the case when the $M_{\chi_{\mathrm{st}}}$ is relatively close in mass to $m_{\tilde{q}}$, up to correction in the light quark masses, there is an enhancement in the SI cross section [107]. Utilizing this effect, for mass splitting of order $30-100 \mathrm{GeV}$, one easily sees detectable size SI cross sections for squark masses that are in accord with LHC limits (see figure 5). At even smaller mass splittings, the models are constrained by XENON. We have verified using micromegas [110] that the small mass splitting between the LSP and the squarks can lead to cross sections of the size we find. In this case the relic density can be brought in accord with WMAP from co-annihilatons. In particular the squarks in the initial state annihilations play a large role in reducing the relic abundance. There is also mixing that derives from rotating between the chiral fermion in the Stueckelberg multiplet. We consider the optimal case where in the mass diagonal basis, the lighter of the two mass eigenstates is the one which couples via the larger mixing. Thus we have taken the mixing in the gaugino stino sector $\cos \theta_{\chi_{\mathrm{st}}} \rightarrow 1$, and have fixed $g_{\mathrm{BL}}=0.65$ in figure 5 . The result of a large scattering cross section does require an LSP above around (500-600) GeV to be consistent with the current limits from the LHC [111-119]. 


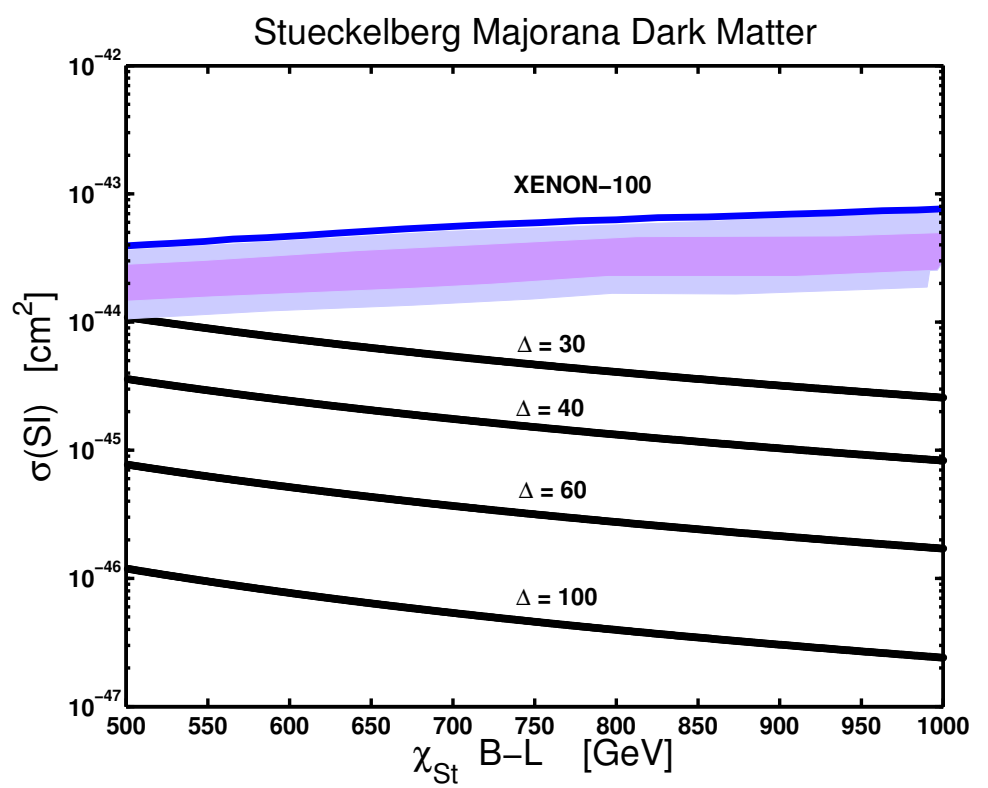

Figure 5. Spin independent $\chi_{\text {st }}$ neutralino-proton cross section vs the Stueckelberg neutralino mass for the case when the Stueckelberg neutralino is the LSP. Exhibited is the spin independent cross section for several combinations of $\Delta \simeq m_{\tilde{q}}-M_{\chi_{\text {st }}}$ (in units of $\mathrm{GeV}$ ). The current limits from XENON-100 are also exhibited.

\subsection{Dirac dark matter}

Additional matter fields in the form of Dirac fermions (and their supersymmetric counter parts, two chiral scalars) can exist in the $\mathrm{U}(1)_{X}$ sector which have only vectorial couplings to the gauge field $X^{\mu}$ and a mass for the Dirac fermions can be generated via terms in the superpotential [45]. As seen already, after mixing of the $B-L$ gauge field $C^{\mu}$ with the field $X^{\mu}$, two mass eigenstates $Z^{\prime}$ and $Z^{\prime \prime}$ arise in the mass diagonal basis each of which have $B-L$ type couplings with the SM fields. In addition, the interaction of the dark sector Dirac field with the $Z^{\prime}, Z^{\prime \prime}$ is given by

$$
\mathcal{L}_{D}=\bar{D} \gamma^{\mu}\left(C_{Z^{\prime} D} Z_{\mu}^{\prime}+C_{Z^{\prime \prime} D} Z_{\mu}^{\prime \prime}\right) D
$$

The interaction vertices with the Dirac particle $(D)$ with the visible sector quarks and leptons enter through the vector mixings so that

$$
C_{Z^{\prime} D}=g_{X} Q_{X} \cos \theta_{\mathrm{BL}}, \quad C_{Z^{\prime \prime} D}=g_{X} Q_{X} \sin \theta_{\mathrm{BL}} .
$$

The dark sector Dirac field can constitute dark matter. It is stable and electrically neutral. Since the model we consider has two components of dark matter, the total relic density $\Omega h^{2}$ will be shared by the neutralino and Dirac particles. In the analysis we assume that the dark matter densities $\varrho_{D}, \varrho_{\chi}$ for the two components in the galaxy are proportional to their respective relic densities such that sum is the total cold dark matter (CDM) density

$$
\frac{\varrho_{D}}{\varrho_{\chi}} \simeq \frac{\Omega_{D}}{\Omega_{\chi}}, \quad \Omega_{\mathrm{CDM}} h^{2}=\underset{\text { (Majorana) }}{\Omega_{\chi} h^{2}}+\underset{\text { (Dirac) }}{\Omega_{D} h^{2}}
$$


The annihilation cross section of $D \bar{D}$ into quarks and leptons via the $Z^{\prime}, Z^{\prime \prime}$ poles is given by

$$
\sigma_{D \bar{D} \rightarrow f \bar{f}}=A_{D, f}\left|P_{Z^{\prime}}-P_{Z^{\prime \prime}}\right|^{2}
$$

where the poles and couplings enter as

$$
\begin{aligned}
P_{V} & =\left(s-M_{V}^{2}+i \Gamma_{V} M_{V}\right)^{-1}, \quad V=\left(Z^{\prime}, Z^{\prime \prime}\right), \\
A_{D, f} & =\frac{g_{D, f}^{2} N_{f}}{48 \pi s}\left(2 M_{D}^{2}+s\right)\left(2 m_{f}^{2}+s\right) \sqrt{\frac{4 m_{f}^{2}-s}{4 M_{D}^{2}-s} \tilde{\Theta}} \\
g_{D, f} & =g_{\mathrm{BL}} Q_{B L, f} g_{X} Q_{X} \sin 2 \theta_{\mathrm{BL}},
\end{aligned}
$$

and where $s=4 M_{D}^{2} /\left(1-v^{2} / 4\right), \tilde{\Theta}=\Theta\left(s-4 m_{f}^{2}\right)$, and $N_{f}=(1,3)$ for (leptons, quarks). The relevant partial $Z^{\prime}, Z^{\prime \prime}$ decay widths were given in table 1 . In addition the $Z^{\prime}, Z^{\prime \prime}$ can decay into the Dirac sector:

$$
\Gamma_{Z^{\prime} \rightarrow D \bar{D}}=\Theta \cdot \frac{M_{Z^{\prime}} g_{D}^{2}}{12 \pi}\left(1+\frac{2 M_{D}^{2}}{M_{Z^{\prime}}^{2}}\right)\left(1-\frac{4 M_{D}^{2}}{M_{Z^{\prime}}^{2}}\right)^{1 / 2}
$$

where $\Theta=\Theta\left(M_{Z^{\prime}}-2 M_{D}\right)$ and $g_{D}=g_{X} Q_{X} \cos \theta_{\mathrm{BL}}$. The partial decay width of the $Z^{\prime \prime}$ is obtained with $M_{Z^{\prime}} \rightarrow M_{Z^{\prime \prime}}$ and $\cos \theta_{\mathrm{BL}} \rightarrow \sin \theta_{\mathrm{BL}}$ in eq. (6.16). The relic density can be calculated by integration over the poles. For the technique of integrating over a pole see [122-124]. The relic density for the 2 components of dark matter can be calculated [45] where for the Dirac component

$$
\Omega_{D} h^{2}=C_{D} J_{D}^{-1}, \quad J_{D}=\int_{0}^{x_{F, D}} \sum_{f}\langle\sigma v\rangle_{D \bar{D} \rightarrow \bar{f} f} d x, \quad C_{D}=2 \times \frac{1.07 \times 10^{9} \mathrm{GeV}^{-1}}{\sqrt{g^{*}} M_{\mathrm{pl}}}(6.17)
$$

In figure 6 we exhibit a satisfaction of the relic density within the WMAP constraint so that

$$
R_{\text {Dirac }}^{\mathrm{St}} \equiv \frac{M_{D}}{M_{Z^{\prime}}} \simeq 1 / 2
$$

where the black bands in figure 6 show a presumed fraction of the the total relic abundance.

Now, unlike the cases studied previously with the Stueckelberg mass growth, here the dark Dirac fermion does not carry a milli-charge and is electrically neutral. The reason the Dirac fermion is neutral is because there is no mixing of the Stueckelberg gauge field with the hypercharge vector boson. Because of its electrical neutrality and unlike a milli charged particle it cannot be stopped by the atmosphere or by dirt and rock in the Earth before it reaches an underground detector. The effective Lagrangian describing the scattering of a dark Dirac fermion from a quark, in the limit of low momentum transfer, is given by $\mathcal{L}_{\text {eff }}=C_{q}^{D} \bar{D} \gamma^{\mu} D \bar{q} \gamma_{\mu} q$. The corresponding spin independent D-proton cross section is

$$
\sigma_{D p}^{\mathrm{SI}}=\frac{\mu_{D p}^{2}}{\pi} G^{2}\left(\frac{1}{M_{Z^{\prime}}^{4}}+\frac{1}{M_{Z^{\prime \prime}}^{4}}-\frac{2}{M_{Z^{\prime}}^{2} M_{Z^{\prime \prime}}^{2}}\right)
$$

where $G=g_{\mathrm{BL}} \sin \theta_{\mathrm{BL}} g_{X} Q_{X} \cos \theta_{\mathrm{BL}}$ and $\mu_{D p}$ is the reduced mass. 

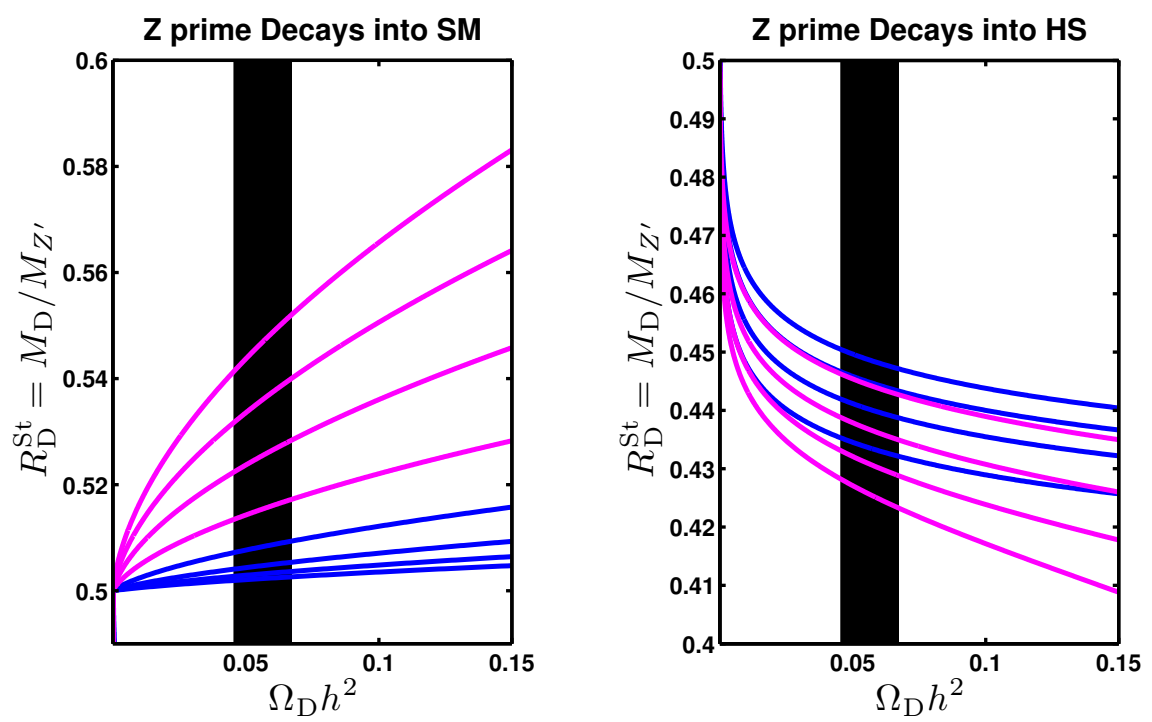

Figure 6. An exhibition of the relic density of the Dirac component of dark matter for various values of $R_{\text {Dirac }}^{\text {St }}$ which is the ratio the Dirac dark matter mass to the Stueckelberg $Z^{\prime}$ mass. The black bands represent about half the relic abundance. For the analysis we fix $g_{\mathrm{BL}}=0.35, g_{X}=$ $0.1, Q_{X}=0.5$. The (blue/darker) curves have the $Z^{\prime}$ mass running in the range $200-500 \mathrm{GeV}$ in steps of $100 \mathrm{GeV}$. We note that for fixed couplings, as $M_{Z^{\prime}}$ gets heavier the curves become more narrow. The (magenta/lighter) curves correspond to $M_{Z^{\prime}}=250 \mathrm{GeV}$ with $\theta_{\mathrm{BL}}=(0.02-0.05)$. Similarly, as $\theta_{\mathrm{BL}}$ becomes progressively smaller for otherwise fixed couplings and fixed $Z^{\prime}$ mass, the curves become more narrow. The right panel is the case when the $Z^{\prime}$ decays mostly into the hidden sector Dirac fermions, i.e., it is the case where $Z^{\prime} \rightarrow D \bar{D}$ is kinematically allowed and in this case the dileptonic signals at the LHC will be depleted. The left panel is the case where $Z^{\prime} \rightarrow D \bar{D}$ is kinematically disallowed and in this case the $Z^{\prime}$ will decay exclusively into the SM particles and thus the dileptonic signal from the process $p p \rightarrow Z^{\prime} \rightarrow l^{+} l^{-}$will be visible.

\begin{tabular}{|ccc|}
\hline $\begin{array}{c}M_{Z^{\prime}} \\
\mathrm{GeV}\end{array}$ & $\begin{array}{c}\sigma_{D p}^{\mathrm{SI}}\left(\theta_{\mathrm{BL}}=(0.03)\right) \\
\mathrm{cm}^{2}\end{array}$ & $\begin{array}{c}\sigma_{D p}^{\mathrm{SI}}\left(\theta_{\mathrm{BL}}=(0.06)\right) \\
\mathrm{cm}^{2}\end{array}$ \\
\hline 200 & $1.9 \times 10^{-44}$ & $7.5 \times 10^{-44}$ \\
300 & $3.7 \times 10^{-45}$ & $1.5 \times 10^{-44}$ \\
400 & $1.2 \times 10^{-45}$ & $4.7 \times 10^{-45}$ \\
500 & $4.8 \times 10^{-46}$ & $1.9 \times 10^{-45}$ \\
\hline
\end{tabular}

Table 2. Approximate values of the spin independent scattering cross section for the Dirac component of dark matter for sample models. The second and third columns have $\theta_{\mathrm{BL}}=(0.03,0.06)$ respectively. The first row is on the edge of the discovery limits from the both XENON and the Tevatron data and is being probed by the LHC. For a given dark matter mass $M_{Z^{\prime}} \sim 2 M_{D}$ in order to satisfy $\left(\Omega h^{2}\right)$ WMAP. Model parameters are otherwise fixed as in figure 4 . The middle column of this table corresponds to the blue/dark curves in figure 7, while the magenta/light region is found to be constrained by the XENON data. Models consistent with the relic density constraint and the XENON constraint are therefore favored if the relic density is satisfied closer to the pole which is obtained for relatively smaller coupling and/or larger $M_{Z^{\prime}}$. 


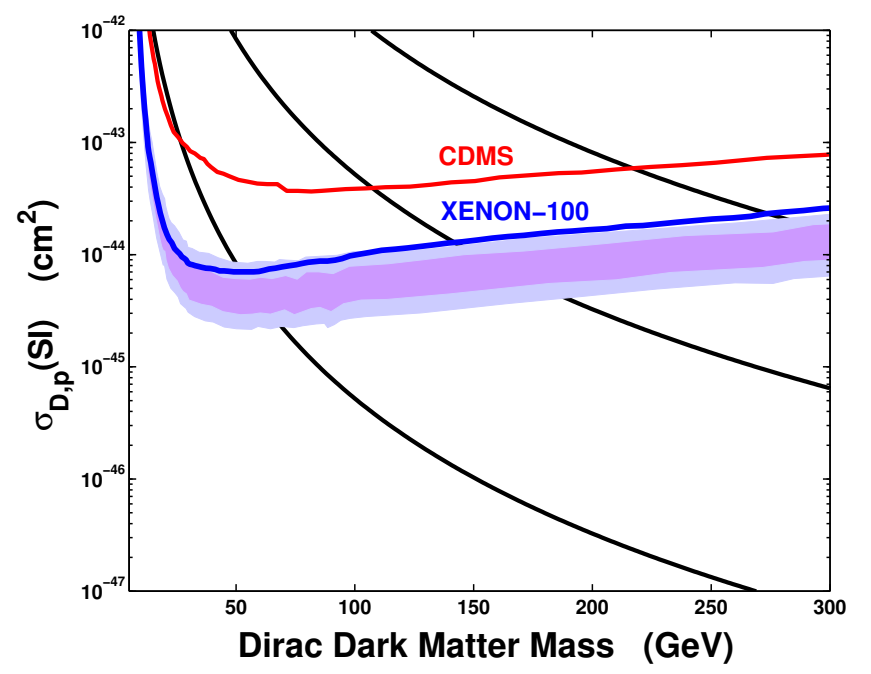

Figure 7. Illustrative curves. At any given point on this plot there exists a funnel where the relic density can be satisfied for perturbative size coupling via the relic density invariant $R_{\text {Dirac }}^{\mathrm{St}}$. The particular values of the parameters on thes curves are $\left(\theta_{\mathrm{BL}}=.001, g_{X}=0.1, Q_{X}=1 / 2\right)$, $\left(\theta_{\mathrm{BL}}=0.01, g_{X}=0.5, Q_{X}=1\right)$, and $\left(\theta_{\mathrm{BL}}=0.05, g_{X}=1 / 2, Q_{X}=1\right)$, where $g_{\mathrm{BL}}=0.35$ and $R_{\text {Dirac }}^{\mathrm{St}} \sim 1 / 2$

Interestingly, for mixing of the size considered in figure $1,\left(\sin \theta_{\mathrm{BL}} \in[0.01,0.05]\right)$ and for natural size couplings $g_{X}=g_{\mathrm{BL}}=O\left(g_{Y}\right)$ and $Q_{X}= \pm 1$ one obtains a spin independent cross sections which are of the size

$$
\sigma_{D p}^{\mathrm{SI}} \sim 10^{-45 \pm 1} \mathrm{~cm}^{2}, \quad M_{Z^{\prime}} \sim(200-300) \mathrm{GeV}
$$

Since $M_{D} \gg m_{p}, \mu_{D p} \sim m_{p}, \sigma_{D p}^{\mathrm{SI}}$ is essentially independent of $M_{D}$. However, compatibility with the WMAP data for the thermal relic density, restricts the ratio $R_{\text {Dirac }}^{S t} \simeq 1 / 2$. Using this constraint the spin independent cross section $\sigma_{D p}^{\mathrm{SI}}$ for the case $M_{Z^{\prime \prime}}^{2} \gg M_{Z^{\prime}}^{2}$ is given by

$$
\sigma_{D p}^{\mathrm{SI}} \simeq \frac{\mu_{D p}^{2}}{\pi} G^{2} \frac{1}{M_{Z^{\prime}}^{4}} \simeq \frac{\mu_{D p}^{2}}{16 \pi} G^{2} \frac{1}{M_{D}^{4}}
$$

which now has a very strong dependence on the Dirac mass. The numerical size of $\sigma_{D p}^{\mathrm{SI}}$ as a function of the Dirac mass is exhibited in figure 5 , and the analysis shows that the $\sigma_{D p}^{\mathrm{SI}}$ predicted by the model is accessible in the XENON experiment. In fact for given values of $g_{\mathrm{BL}}, \theta_{\mathrm{BL}}, g_{X} Q_{X}$ the current limits from XENON100 already put lower limits on the Dirac mass. We can also use the current upper limit on $\sigma^{\mathrm{SI}}$ from the XENON100 experiment which gives $\sigma^{\mathrm{SI}}=7 \times 10^{-45} \mathrm{~cm}^{2}$ for a WIMP mass of $50 \mathrm{GeV}$, to put a general constraint on $|G| / M_{D}^{2}$ so that

$$
|G| / M_{D}^{2} \lesssim 3 \times 10^{-8} \quad\left(M_{D} \text { in } \mathrm{GeV}\right)
$$

We note again that the preceding analysis is very different from the previous Stueckelberg analyses where the Dirac fermion in the hidden sector develops a milli charge. As 
already pointed out this arises in models where one mixes the Stueckelberg gauge boson with the hypercharge gauge field. In this case the scattering of the Dirac fermion from a quark will have not only the $Z^{\prime}$ pole in the t-channel but also a $Z$ boson pole and a photon pole as well. In the present model the $Z$ and the photon pole are both absent. The Dirac dark matter candidate is electrically neutral.

As mentioned earlier, for $M_{Z^{\prime}} \sim 2 M_{D}$, the relic density will always be satisfied for perturbative size couplings. For $M_{Z^{\prime}}<2 M_{D}$ but close to $2 M_{D}$ the $Z^{\prime}$ signal will manifest at colliders and the relic density can also be satisfied. However, for the case $M_{Z^{\prime}}>$ $2 M_{D}$, while the relic density can be satisfied, the $Z^{\prime}$ signal becomes suppressed due to the branching ratio into the hidden sector overtaking the branching ratio in the visible sector in the presence of mass and kinetic mixings [32, 125, 126]. In addition, the Breit-Wigner enhancement of the annihilation of Dirac particles in the halo [46] can be operative very close to the pole and the following three possibilities become simultaneous observables:

1. Observation of a very light and narrow $Z^{\prime}$ vector boson in the dilepton channel at the LHC (see also [10]).

2. Observation of the flux of positrons via Satellite data (PAMELA/FERMI) [127, 128] from the Breit-Wigner Enhancement in the dark matter annihilations in the galactic halo [46] consistent with WMAP data [60].

3. Relic abundance of dark matter split between a neutralino and dark Dirac (see also [45]) .

4. Observational prospects for the corresponding Dark Dirac component in direct detections experiments such as XENON (analyzed here for the neutral dark Dirac particle via the Stueckelberg mechanism).

Let us add, that just recently, the $730 \mathrm{~kg}$ days of the CRESST-II Dark Matter Search was released [129]. Two preferred regions are reported on, and one such region appears close to the CoGeNT preferred region [130]. Very low mass neutralino dark matter with MSSM field content and cross sections of the size needed to explain the CoGeNT are not consistent with the collider constraints [131-135]. This result has been confirmed by the LHC with its updated constraints on the SUSY Higgs sector [136], wherein large tan $\beta$ and low mass SUSY Higgs of the size needed to explain the spin-independant scattering are further excluded. The preferred region reported by CRESST-II with heavier dark matter mass may be accommodated for a thermal relic with relic density satisfied via the Z-pole in the MSSM. Such could arise with non-universal gaugino masses at the the high-scale (see $[116,117])$ leading to WIMP masses close to $45 \mathrm{GeV}$. The far boundary of the CRESSTII $2 \sigma$ region terminating close to $55 \mathrm{GeV}$ may also be achieved with relic density satisfied via the Higgs pole (see the analysis of [137]). A dedicated analyses with the new constraints on the SUSY Higgs sector from the LHC [136] would be needed to make a more definitive statement - however the CRESST-II results at these potential dark mater masses do not correspond to reported event rates with CDMS or XENON [61,62]. The extended model class we discuss can produce spin independent cross sections with larger cross sections than that of the MSSM via the Dirac component of Dark Matter (see figure 7). 


\section{Discriminating Stueckelberg from models with spontaneous breaking}

One may discriminate between the Stueckelberg mass growth for a $B-L$ gauge boson in the models discussed here and other models where the mass growth for the $B-L$ gauge boson occurs by spontaneous breaking. In the above, we have already discussed the mass growth of a $B-L$ gauge boson by the Stueckelberg mechanism. For the case when the mass growth occurs via spontaneous breaking there are two possibilities: (i) spontaneous symmetry breaking of $\mathrm{U}(1)_{B-L}$ occurs violating R-parity invariance, (ii) spontaneous symmetry breaking of $\mathrm{U}(1)_{B-L}$ occurs without violating R-parity invariance. We discuss these two cases below individually.

\subsection{Spontaneous symmetry breaking of $B-L$ and R-parity violation}

The simplest example of this is when we consider the superpotential of eq. (3.5). Let us assume that the potential of the $\tilde{\nu}^{c}$ field is such that it develops a VEV. In this case one will have a spontaneous breaking of not only $B-L$ but also of R-parity as indicated by the term $L H_{u}\left\langle\tilde{\nu}^{c}\right\rangle$ in eq. (3.5) after $\tilde{\nu}^{c}$ develops a VEV. In the mass diagonal basis it will lead to other R-parity violating terms, i.e., $L L e^{c}$ and $Q L d^{c}$. Here the LSP is no longer stable and specifically the neutralino cannot be a dark matter particle. Further, since the neutralino is not stable, the signals of supersymmetry for this case will be very different at hadron colliders. Specifically if the neutralino decays inside the detector, there will be no missing energy signatures which are the typical hallmarks of supersymmetry signatures with R-parity symmetry (For a review see [141]). Further, for the case when there is a spontaneous breaking of R-parity symmetry via the VEV growth of the right handed sneutrino, there will be D term contributions to the slepton squared masses proportional to $g_{\mathrm{BL}}^{2}\left\langle\tilde{\nu}^{c}\right\rangle^{2}[12]$. Such terms are absent for the case when the mass growth for the $B-L$ gauge boson occurs preserving R-parity invariance as discussed in 7.2.

\section{2 $B-L$ models for R-parity conservation}

We further consider now the possibility that $B-L$ symmetry is broken but a residual $\mathrm{R}$ parity symmetry still persists. This is indeed possible following the general line of reasoning of [138] (see also [139]). Thus consider additional fields in the theory such as a vector like multiplet which has the $\mathrm{SU}(3)_{C} \otimes \mathrm{SU}(2)_{L} \otimes \mathrm{U}(1)_{Y} \otimes \mathrm{U}(1)_{B-L}$ quantum numbers as follows

$$
\Phi \sim\left(1,1,0,-Q_{\mathrm{BL}}\right), \quad \bar{\Phi} \sim\left(1,1,0, Q_{\mathrm{BL}}\right) .
$$

Let us suppose that one manufactures a potential so that VEV formation for the fields $\Phi$ and $\bar{\Phi}$ occurs. In this case $B-L$ will be broken. However, as long as $3(B-L)$ is an even integer R-parity will be preserved. This means that the residual theory will have a $Z_{2}$ R-parity symmetry. Thus, for example, the VEV formation of a scalar field with $3(B-L)= \pm 2$ will violate $B-L$ but preserve R-parity. In the process of the mass mass growth of the $B-L$ gauge boson, one combination of the imaginary parts of $\Phi^{0}$ and $\bar{\Phi}^{0}$ will be absorbed while there would three spin zero fields: $2 \mathrm{CP}$ even and one $\mathrm{CP}$ odd (the part orthogonal to the imaginary parts of $\Phi^{0}$ and $\bar{\Phi}^{0}$ which is absorbed) Higgs field. In contrast for the $\mathrm{U}(1)_{B-L} \otimes \mathrm{U}(1)_{X}$ model discussed here, one is left with only two additional 
scalars, $\rho_{X}, \rho_{\mathrm{BL}}$, or $\rho, \rho^{\prime}$, which are both $\mathrm{CP}$ even. Specifically there is no additional CP odd Higgs boson for the Stueckelberg models. So this provides a discrimination between the two models.

There are several interesting and distinguishing features between the $\mathrm{U}(1)_{B-L} \otimes \mathrm{U}(1)_{X}$ model and the $\mathrm{U}(1)_{B-L}$ model. This difference can be seen by comparing eq. (4.10) vs eq. (5.7). Thus in eq. (4.10) one finds that the mass growth of a $B-L$ gauge boson by spontaneous breaking or by the Stueckelberg mechanism would require the gauge boson to be very heavy. Thus for $g_{\mathrm{BL}} \sim 1$, one will typically have a mass of the $B-L$ gauge boson to be greater than $\sim 6 \mathrm{TeV}[86,140]$. In contrast, from eqs. (5.7) we find that in the $\mathrm{U}(1)_{X} \otimes \mathrm{U}(1)_{B-L}$ model, there are two extra massive gauge bosons beyond what one has in the Standard Model. Thus the heavier one, i.e., the $Z^{\prime \prime}$ gauge boson, is indeed several $\mathrm{TeV}$ in mass. However, the $Z^{\prime}$ boson we discuss can be much lighter, and can lie in the few hundred $\mathrm{GeV}$ range. Thus the observation of a low lying $Z^{\prime}$ with decay branching ratios characteristic of a $B-L$ gauge boson will be a clear indication of the Stueckelberg model involving mixing of $\mathrm{U}(1)_{X}$ and $\mathrm{U}(1)_{B-L}$ discussed here.

\section{Conclusion}

In this work we have proposed the Stueckelberg mechanism for the mass growth of a $B-L$ gauge boson. It was then shown that under the constraints of charge conservation and the absence of a Fayet-Iliopoulos D term, that R-parity cannot be spontaneously broken in the minimal model of radiative electroweak symmetry breaking. The above is in contrast to models where the mass of the $B-L$ gauge boson is generated by the Higgs mechanism through the VEV formation for the field $\tilde{\nu}^{c}$ which breaks R-parity.

A comparison to the case where the $B-L$ symmetry is spontaneously broken but the R-parity symmetry is preserved was also given and its distinguishing features from the Stueckelberg mass growth for the $B-L$ gauge boson are uncovered. Further, we analyzed a $\mathrm{U}(1)_{X} \otimes \mathrm{U}(1)_{B-L}$ Stueckelberg extension of MSSM where a massive $Z^{\prime}$ boson with $B-L$ interactions can lie in the sub $\mathrm{TeV}$ region, i.e, $M_{Z^{\prime}}<1 \mathrm{TeV}$. The observation of a $Z^{\prime}$ in the sub $\mathrm{TeV}$ region with $B-L$ quantum numbers deduced via branching ratios into charged leptons will provide a test of the $\mathrm{U}(1)_{X} \otimes \mathrm{U}(1)_{B-L}$ Stueckelberg extension discussed here.

Other tests of the proposed Stueckelberg models were also discussed. This includes an analysis of the production and decay of the Stueckelberg spin 0 boson $\rho$ which has only loop decays into SM final states via sfermion loops. An interesting decay of the $\rho$ is into $\gamma \gamma$ was analyzed and shown to have the possibility of observation at the LHC with $\sqrt{s}=14 \mathrm{TeV}$.

With hidden sector Dirac fermions in the $\mathrm{U}(1)_{X} \otimes \mathrm{U}(1)_{B-L}$ Stueckelberg extension, two component dark matter manifests, with one component being either the MSSM neutralino or the Stueckelberg neutralino and the other component being a neutral Dirac fermion. An analysis of the relic density for the Stueckelberg neutralino and the Stueckelberg neutralinoproton spin independent cross section were also discussed. An analysis of the second dark matter component consisting of the Dirac fermion as dark matter was also given and it was shown that the current XENON100 data already puts constraints on the Dirac fermion mass and mixing angles. The constraints from the XENON100 data and the LHC data on 
the couplings of the $Z^{\prime}$ boson and dark Dirac fermion were shown to be comparable, both of which limit the mixing of the $B-L$ and dark sector. Thus the proposed model produces LHC and dark matter signals at mass scales that are accessible to such experiments and will be tested further as the new data comes in.

\section{Acknowledgments}

P.F.P. would like to thank Northeastern University for hospitality in the beginning of this project. The work of D.F. is supported by DOE DE-FG02-95ER40899 and by the Michigan Center for Theoretical Physics. The work of P. F. P. is supported by the James Arthur Fellowship at CCPP-New York University. The work of P.N. is supported in part by NSF grant PHY-0757959 and PHY-0704067. D.F. would like to thank CERN Theory Group for their hospitality while this work was nearing completion.

Open Access. This article is distributed under the terms of the Creative Commons Attribution Noncommercial License which permits any noncommercial use, distribution, and reproduction in any medium, provided the original author(s) and source are credited.

\section{References}

[1] P. Nath and P. Fileviez Perez, Proton stability in grand unified theories, in strings and in branes, Phys. Rept. 441 (2007) 191 [hep-ph/0601023] [InSPIRE].

[2] A.H. Chamseddine, R.L. Arnowitt and P. Nath, Locally supersymmetric grand unification, Phys. Rev. Lett. 49 (1982) 970 [InSPIRE].

[3] R.L. Arnowitt and P. Nath, SUSY mass spectrum in SU(5) supergravity grand unification, Phys. Rev. Lett. 69 (1992) 725 [INSPIRE].

[4] P. Nath and R.L. Arnowitt, Radiative breaking, proton stability and the viability of no scale supergravity models, Phys. Lett. B 287 (1992) 89 [INSPIRE].

[5] P. Nath, Twenty years of SUGRA, hep-ph/0307123 [INSPIRE].

[6] H. Goldberg, Constraint on the photino mass from cosmology, Phys. Rev. Lett. 50 (1983) 1419 [Erratum ibid. 103 (2009) 099905] [INSPIRE].

[7] B. Körs and P. Nath, A Stueckelberg extension of the standard model, Phys. Lett. B 586 (2004) 366 [hep-ph/0402047] [INSPIRE].

[8] B. Körs and P. Nath, A supersymmetric Stueckelberg U(1) extension of the MSSM, JHEP 12 (2004) 005 [hep-ph/0406167] [INSPIRE].

[9] B. Körs and P. Nath, Aspects of the Stueckelberg extension, JHEP 07 (2005) 069 [hep-ph/0503208] [INSPIRE].

[10] D. Feldman, Z. Liu and P. Nath, Probing a very narrow $Z^{\prime}$ boson with CDF and Do data, Phys. Rev. Lett. 97 (2006) 021801 [hep-ph/0603039] [INSPIRE].

[11] D. Feldman, Z. Liu and P. Nath, The Stueckelberg Z Prime at the LHC: discovery potential, signature spaces and model discrimination, JHEP 11 (2006) 007 [hep-ph/0606294] [INSPIRE]. 
[12] P. Fileviez Perez and S. Spinner, Spontaneous R-parity breaking and left-right symmetry, Phys. Lett. B 673 (2009) 251 [arXiv:0811.3424] [INSPIRE].

[13] V. Barger, P. Fileviez Perez and S. Spinner, Minimal gauged U(1) $)_{B-L}$ model with spontaneous R-parity violation, Phys. Rev. Lett. 102 (2009) 181802 [arXiv:0812.3661] [INSPIRE].

[14] P. Fileviez Perez and S. Spinner, Spontaneous R-parity breaking in SUSY models, Phys. Rev. D 80 (2009) 015004 [arXiv:0904.2213] [InSPIRE].

[15] L.L. Everett, P. Fileviez Perez and S. Spinner, The right side of TeV scale spontaneous R-parity violation, Phys. Rev. D 80 (2009) 055007 [arXiv: 0906.4095] [INSPIRE].

[16] P. Fileviez Perez and S. Spinner, The fate of R-parity, Phys. Rev. D 83 (2011) 035004 [arXiv:1005.4930] [INSPIRE].

[17] P. Fileviez Perez, S. Spinner and M.K. Trenkel, The LSP stability and new Higgs signals at the LHC, Phys. Rev. D 84 (2011) 095028 [arXiv:1103.5504] [inSPIRE].

[18] S.P. Martin, Some simple criteria for gauged R-parity, Phys. Rev. D 46 (1992) 2769 [hep-ph/9207218] [INSPIRE].

[19] S. Khalil and A. Masiero, Radiative B-L symmetry breaking in supersymmetric models, Phys. Lett. B 665 (2008) 374 [arXiv:0710.3525] [INSPIRE].

[20] M. Frank, Phenomenology of left-right supersymmetric models with broken B-L symmetry, Phys. Rev. D 63 (2001) 034009 [inSPIRE].

[21] C. Aulakh and R.N. Mohapatra, Neutrino as the supersymmetric partner of the Majoron, Phys. Lett. B 119 (1982) 136 [INSPIRE].

[22] L.J. Hall and M. Suzuki, Explicit R-parity breaking in supersymmetric models, Nucl. Phys. B 231 (1984) 419 [inSPIRE].

[23] A. Masiero and J. Valle, A model for spontaneous R-parity breaking, Phys. Lett. B 251 (1990) 273 [INSPIRE].

[24] R. Mohapatra, New contributions to neutrinoless double $\beta$ decay in supersymmetric theories, Phys. Rev. D 34 (1986) 3457 [InSPIRE].

[25] A. Font, L.E. Ibáñez and F. Quevedo, Does proton stability imply the existence of an extra Z0?, Phys. Lett. B 228 (1989) 79 [INSPIRE].

[26] E. Accomando, A. Belyaev, L. Fedeli, S.F. King and C. Shepherd-Themistocleous, $Z^{\prime}$ physics with early LHC data, Phys. Rev. D 83 (2011) 075012 [arXiv:1010.6058] [INSPIRE].

[27] L. Basso, Phenomenology of the minimal B-L extension of the standard model at the LHC, arXiv: 1106.4462 [INSPIRE].

[28] G.M. Pruna, Phenomenology of the minimal B - L model: the Higgs sector at the Large Hadron Collider and future Linear Colliders, arXiv:1106.4691 [INSPIRE].

[29] L. Basso, A. Belyaev, S. Moretti, G.M. Pruna and C.H. Shepherd-Themistocleous, $Z_{B-L}^{\prime}$ discovery potential at the LHC for $\sqrt{s}=7 \mathrm{TeV}$, PoS(ICHEP2010) 381 [arXiv:1011.0872] [INSPIRE].

[30] D. Feldman, B. Körs and P. Nath, Extra-weakly interacting dark matter, Phys. Rev. D 75 (2007) 023503 [hep-ph/0610133] [INSPIRE]. 
[31] K. Cheung and T.-C. Yuan, Hidden fermion as milli-charged dark matter in Stueckelberg $Z^{\prime}$ model, JHEP 03 (2007) 120 [hep-ph/0701107] [INSPIRE].

[32] D. Feldman, Z. Liu and P. Nath, The Stueckelberg Z' extension with kinetic mixing and milli-charged dark matter from the hidden sector, Phys. Rev. D 75 (2007) 115001 [hep-ph/0702123] [INSPIRE].

[33] D. Feldman, Z. Liu and P. Nath, Recent developments in supersymmetric and hidden sector dark matter, AIP Conf. Proc. 1078 (2009) 116 [arXiv:0806.4683] [InSPIRE].

[34] D. Feldman, Z. Liu and P. Nath, The Stueckelberg extension and milli weak and milli charged dark matter, AIP Conf. Proc. 939 (2007) 50 [arXiv:0705.2924] [InSPIRE].

[35] M. Pospelov, A. Ritz and M.B. Voloshin, Secluded WIMP dark matter, Phys. Lett. B 662 (2008) 53 [arXiv:0711.4866] [INSPIRE].

[36] J.L. Feng and J. Kumar, The WIMPless miracle: dark-matter particles without weak-scale masses or weak interactions, Phys. Rev. Lett. 101 (2008) 231301 [arXiv:0803.4196] [INSPIRE].

[37] A. Ibarra, A. Ringwald and C. Weniger, Hidden gauginos of an unbroken $\mathrm{U}(1)$ : cosmological constraints and phenomenological prospects, JCAP 01 (2009) 003 [arXiv: 0809.3196] [INSPIRE].

[38] Y. Cui, D.E. Morrissey, D. Poland and L. Randall, Candidates for inelastic dark matter, JHEP 05 (2009) 076 [arXiv:0901.0557] [INSPIRE].

[39] C. Cheung, J.T. Ruderman, L.-T. Wang and I. Yavin, Kinetic mixing as the origin of light dark scales, Phys. Rev. D 80 (2009) 035008 [arXiv: 0902.3246] [INSPIRE].

[40] S. Gopalakrishna, S.J. Lee and J.D. Wells, Dark matter and Higgs boson collider implications of fermions in an abelian-gauged hidden sector, Phys. Lett. B 680 (2009) 88 [arXiv:0904.2007] [INSPIRE].

[41] K. Cheung, K.-H. Tsao and T.-C. Yuan, Hidden sector dirac dark matter, stueckelberg $Z^{\prime}$ model and the CDMS and XENON experiments, arXiv:1003.4611 [INSPIRE].

[42] P. Nath, Predicted signatures at the LHC from U(1) extensions of the standard model, Mod. Phys. Lett. A 25 (2010) 3003 [arXiv:0812.0958] [inSPIRE].

[43] Z. Liu, Hidden sector models and signatures, Nucl. Phys. Proc. Suppl. 200-202 (2010) 133 [arXiv:0910.0061] [INSPIRE].

[44] P. Nath et al., The hunt for new physics at the Large Hadron Collider, Nucl. Phys. Proc. Suppl. 200-202 (2010) 185 [arXiv:1001.2693] [INSPIRE].

[45] D. Feldman, Z. Liu, P. Nath and G. Peim, Multicomponent dark matter in supersymmetric hidden sector extensions, Phys. Rev. D 81 (2010) 095017 [arXiv: 1004.0649] [INSPIRE].

[46] D. Feldman, Z. Liu and P. Nath, PAMELA positron excess as a signal from the hidden sector, Phys. Rev. D 79 (2009) 063509 [arXiv:0810.5762] [INSPIRE].

[47] N. Arkani-Hamed, D.P. Finkbeiner, T.R. Slatyer and N. Weiner, A theory of dark matter, Phys. Rev. D 79 (2009) 015014 [arXiv:0810.0713] [INSPIRE].

[48] D. Feldman, Z. Liu, P. Nath and B.D. Nelson, Explaining PAMELA and WMAP data through coannihilations in extended SUGRA with collider implications,

Phys. Rev. D 80 (2009) 075001 [arXiv:0907.5392] [INSPIRE]. 
[49] N. Chen, D. Feldman, Z. Liu, P. Nath and G. Peim, Higgsino dark matter model consistent with galactic cosmic ray data and possibility of discovery at LHC-7,

Phys. Rev. D 83 (2011) 023506 [arXiv: 1010.0939] [InSPIRE].

[50] N. Chen, D. Feldman, Z. Liu, P. Nath and G. Peim, Low mass gluino within the sparticle landscape, implications for dark matter and early discovery prospects at LHC-7,

Phys. Rev. D 83 (2011) 035005 [arXiv: 1011.1246] [InSPIRE].

[51] D. Feldman, Superparticle signatures: from PAMELA to the LHC, Nucl. Phys. Proc. Suppl. 200-202 (2010) 82 [arXiv:0908.3727] [InSPIRE].

[52] A. Arvanitaki, N. Craig, S. Dimopoulos, S. Dubovsky and J. March-Russell, String photini at the LHC, Phys. Rev. D 81 (2010) 075018 [arXiv:0909.5440] [inSPIRE].

[53] M. Ahlers, J. Jaeckel, J. Redondo and A. Ringwald, Probing hidden sector photons through the Higgs window, Phys. Rev. D 78 (2008) 075005 [arXiv:0807.4143] [InSPIRE].

[54] M. Goodsell, J. Jaeckel, J. Redondo and A. Ringwald, Naturally light hidden photons in LARGE volume string compactifications, JHEP 11 (2009) 027 [arXiv:0909.0515] [INSPIRE].

[55] J. Jaeckel and A. Ringwald, The low-energy frontier of particle physics, Ann. Rev. Nucl. Part. Sci. 60 (2010) 405 [arXiv: 1002.0329] [INSPIRE].

[56] M. Williams, C. Burgess, A. Maharana and F. Quevedo, New constraints (and motivations) for abelian gauge bosons in the MeV-TeV mass range, arXiv:1103.4556 [INSPIRE].

[57] Y. Mambrini, The $Z Z^{\prime}$ kinetic mixing in the light of the recent direct and indirect dark matter searches, JCAP 07 (2011) 009 [arXiv:1104.4799] [INSPIRE].

[58] A.E. Nelson and J. Scholtz, Dark light, dark matter and the misalignment mechanism, Phys. Rev. D 84 (2011) 103501 [arXiv:1105.2812] [INSPIRE].

[59] Y. Mambrini, Specific Dark Matter signatures from hidden U(1), PoS (QFTHEP2010) 027 [arXiv: 1012.0447] [INSPIRE].

[60] WMAP collaboration, E. Komatsu et al., Seven-year Wilkinson Microwave Anisotropy Probe (WMAP) observations: cosmological interpretation, Astrophys. J. Suppl. 192 (2011) 18 [arXiv:1001.4538] [INSPIRE].

[61] XENON100 collaboration, E. Aprile et al., Dark matter results from 100 live days of XENON100 data, Phys. Rev. Lett. 107 (2011) 131302 [arXiv:1104.2549] [INSPIRE].

[62] CDMS-II collaboration, Z. Ahmed et al., Dark matter search results from the CDMS II experiment, Science 327 (2010) 1619 [arXiv:0912.3592] [INSPIRE].

[63] S. Abel, M. Goodsell, J. Jaeckel, V. Khoze and A. Ringwald, Kinetic mixing of the photon with hidden $\mathrm{U}(1) s$ in string phenomenology, JHEP 07 (2008) 124 [arXiv:0803.1449] [INSPIRE].

[64] Y. Zhang, $Z^{\prime}$ mixing effect in Stueckelberg extended effective theory, Chin. Phys. Lett. 26 (2009) 081102.

[65] Y. Zhang, $Z^{\prime}$ phenomenology: a model-independent analysis and fit in combination of chiral effective theory and anomaly cancellation, arXiv:1106.0163 [INSPIRE].

[66] Y. Zhang and Q. Wang, Parameterization of general $Z-\gamma-Z^{\prime}$ mixings in an electroweak chiral theory, arXiv: 1011.4418 [INSPIRE]. 
[67] C. Burgess et al., Continuous global symmetries and hyperweak interactions in string compactifications, JHEP 07 (2008) 073 [arXiv: 0805.4037] [INSPIRE].

[68] J. Heeck and W. Rodejohann, Kinetic and mass mixing with three abelian groups, Phys. Lett. B 705 (2011) 369 [arXiv:1109.1508] [inSPIRE].

[69] C. Corianò, M. Guzzi, N. Irges and A. Mariano, Axion and neutralinos from supersymmetric extensions of the standard model with anomalous $\mathrm{U}(1)$ 's, Phys. Lett. B 671 (2009) 87 [arXiv:0811.0117] [INSPIRE].

[70] C. Corianò, M. Guzzi, A. Mariano and S. Morelli, A light supersymmetric axion in an anomalous abelian extension of the standard model, Phys. Rev. D 80 (2009) 035006 [arXiv: 0811. 3675] [INSPIRE].

[71] C. Corianò, M. Guzzi, G. Lazarides and A. Mariano, Cosmological properties of a gauged axion, Phys. Rev. D 82 (2010) 065013 [arXiv:1005.5441] [INSPIRE].

[72] P. Anastasopoulos et al., Minimal anomalous $\mathrm{U}(1)^{\prime}$ extension of the MSSM, Phys. Rev. D 78 (2008) 085014 [arXiv:0804.1156] [InSPIRE].

[73] T. Aoyama, M. Hayakawa, T. Kinoshita and M. Nio, Dark matter in anomalous U(1)' models with neutral mixing, Phys. Rev. D 84 (2011) 051702 [arXiv:1105.4753] [INSPIRE].

[74] F. Fucito, A. Lionetto, A. Mammarella and A. Racioppi, Stueckelino dark matter in anomalous U(1)' models, Eur. Phys. J. C 69 (2010) 455 [arXiv:0811.1953] [InSPIRE].

[75] G. Panotopoulos and P. Tuzon, The physics of a new gauge boson in a Stueckelberg extension of the two-Higgs-doublet model, JHEP 07 (2011) 039 [arXiv:1102.5726] [INSPIRE].

[76] P.G. Camara, L.E. Ibáñez and F. Marchesano, RR photons, JHEP 09 (2011) 110 [arXiv:1106.0060] [INSPIRE].

[77] M.J. Dolan, J. Marsano, N. Saulina and S. Schäfer-Nameki, F-theory GUTs with U(1) symmetries: generalities and survey, Phys. Rev. D 84 (2011) 066008 [arXiv:1102.0290] [INSPIRE].

[78] M. Cvetič, J. Halverson and P. Langacker, Implications of string constraints for exotic matter and $Z^{\prime} s$ beyond the standard model, JHEP 11 (2011) 058 [arXiv:1108.5187] [INSPIRE].

[79] M. Kalb and P. Ramond, Classical direct interstring action, Phys. Rev. D 9 (1974) 2273 [INSPIRE].

[80] P. Fayet and J. Iliopoulos, Spontaneously broken supergauge symmetries and goldstone spinors, Phys. Lett. B 51 (1974) 461 [INSPIRE].

[81] S.P. Martin and M.T. Vaughn, Two loop renormalization group equations for soft supersymmetry breaking couplings, Phys. Rev. D 50 (1994) 2282 [Erratum ibid. D 78 (2008) 039903] [hep-ph/9311340] [INSPIRE].

[82] P. Nath and R.L. Arnowitt, Nonuniversal soft SUSY breaking and dark matter, Phys. Rev. D 56 (1997) 2820 [hep-ph/9701301] [INSPIRE].

[83] M. Ambroso and B. Ovrut, The B-L/electroweak hierarchy in heterotic string and M-theory, JHEP 10 (2009) 011 [arXiv: 0904.4509] [InSPIRE].

[84] M. Ambroso and B.A. Ovrut, The B-L/electroweak hierarchy in smooth heterotic compactifications, Int. J. Mod. Phys. A 25 (2010) 2631 [arXiv:0910.1129] [InSPIRE]. 
[85] M. Ambroso and B.A. Ovrut, The mass spectra, hierarchy and cosmology of B-L MSSM heterotic compactifications, arXiv:1005.5392 [INSPIRE].

[86] M.S. Carena, A. Daleo, B.A. Dobrescu and T.M. Tait, $Z^{\prime}$ gauge bosons at the Tevatron, Phys. Rev. D 70 (2004) 093009 [hep-ph/0408098] [INSPIRE].

[87] Muon G-2 collaboration, G.W. Bennett et al., Measurement of the negative muon anomalous magnetic moment to 0.7 ppm, Phys. Rev. Lett. 92 (2004) 161802 [hep-ex/0401008] [INSPIRE].

[88] D0 collaboration, V.M. Abazov et al., Search for a heavy neutral gauge boson in the dielectron channel with $5.4 \mathrm{fb}^{-1}$ of ppbar collisions at $\sqrt{\mathrm{s}}=1.96 \mathrm{TeV}$, Phys. Lett. B 695 (2011) 88 [arXiv: 1008. 2023] [INSPIRE].

[89] T. Sjöstrand, S. Mrenna and P.Z. Skands, PYTHIA 6.4 physics and manual, JHEP 05 (2006) 026 [hep-ph/0603175] [INSPIRE].

[90] J. Conway et al., PGS4 - Pretty Good Simulation of high energy collision, http://physics.ucdavis.edu/ ${ }^{\sim}$ conway/research/software/pgs/pgs4-general.htm.

[91] G. Aad et al., Search for dilepton resonances in pp collisions at $\sqrt{s}=7 \mathrm{TeV}$ with the ATLAS detector, arXiv:1108.1582 [INSPIRE].

[92] ATLAS collaboration, Search for high mass dilepton resonances in pp collisions at $\sqrt{s}=7 \mathrm{TeV}$ with the ATLAS experiment, ATLAS-CONF-2011-083 (2011).

[93] E. Salvioni, G. Villadoro and F. Zwirner, Minimal $Z^{\prime}$ models: present bounds and early LHC reach, JHEP 11 (2009) 068 [arXiv:0909.1320] [INSPIRE].

[94] M.S. Chanowitz, A heavy little higgs and a light $Z^{\prime}$ under the radar, Phys. Rev. D 84 (2011) 035014 [arXiv:1102.3672] [InSPIRE].

[95] CMS collaboration, S. Chatrchyan et al., Search for resonances in the dilepton mass distribution in pp collisions at $\sqrt{s}=7 \mathrm{TeV}$, JHEP 05 (2011) 093 [arXiv:1103.0981] [INSPIRE].

[96] A. Djouadi, Squark effects on Higgs boson production and decay at the LHC, Phys. Lett. B 435 (1998) 101 [hep-ph/9806315] [INSPIRE].

[97] M. Spira, QCD effects in Higgs physics, Fortsch. Phys. 46 (1998) 203 [hep-ph/9705337] [INSPIRE].

[98] G.F. Giudice, R. Rattazzi and J.D. Wells, Graviscalars from higher dimensional metrics and curvature Higgs mixing, Nucl. Phys. B 595 (2001) 250 [hep-ph/0002178] [INSPIRE].

[99] A. De Rujula, J. Lykken, M. Pierini, C. Rogan and M. Spiropulu, Higgs look-alikes at the LHC, Phys. Rev. D 82 (2010) 013003 [arXiv:1001.5300] [INSPIRE].

[100] I. Low, J. Lykken and G. Shaughnessy, Singlet scalars as Higgs imposters at the Large Hadron Collider, Phys. Rev. D 84 (2011) 035027 [arXiv: 1105.4587] [InSPIRE].

[101] P.J. Fox, D. Tucker-Smith and N. Weiner, Higgs friends and counterfeits at hadron colliders, JHEP 06 (2011) 127 [arXiv:1104.5450] [INSPIRE].

[102] International Europhysics Conference on High Energy Physics (EPS-HEP2011), July 21-27, Grenoble, France (2011).

[103] XXV International Symposium on Lepton Photon Interactions at High Energies (Lepton Photon 11), August 22-27, Mumbai, India (2011). 
[104] D. Feldman and G. Kane, A Wino-like LSP world: theoretical and phenomenological motivations, in Perspectives on Supersymmetry II, G.K. Kane ed., World Scientific, Singapore (2010).

[105] M. Drees and M. Nojiri, Neutralino-nucleon scattering revisited, Phys. Rev. D 48 (1993) 3483 [hep-ph/9307208] [INSPIRE].

[106] M. Drees and M.M. Nojiri, New contributions to coherent neutralino-nucleus scattering, Phys. Rev. D 47 (1993) 4226 [hep-ph/9210272] [INSPIRE].

[107] J. Hisano, K. Ishiwata and N. Nagata, Gluon contribution to the dark matter direct detection, Phys. Rev. D 82 (2010) 115007 [arXiv:1007.2601] [INSPIRE].

[108] U. Chattopadhyay, T. Ibrahim and P. Nath, Effects of CP-violation on event rates in the direct detection of dark matter, Phys. Rev. D 60 (1999) 063505 [hep-ph/9811362] [INSPIRE].

[109] A. Corsetti and P. Nath, Gaugino mass nonuniversality and dark matter in SUGRA, strings and D-brane models, Phys. Rev. D 64 (2001) 125010 [hep-ph/0003186] [INSPIRE].

[110] G. Bélanger, F. Boudjema, A. Pukhov and A. Semenov, Dark matter direct detection rate in a generic model with micrOMEGAs 2.2, Comput. Phys. Commun. 180 (2009) 747 [arXiv:0803.2360] [INSPIRE].

[111] ATLAS collaboration, G. Aad et al., Search for supersymmetry using final states with one lepton, jets and missing transverse momentum with the ATLAS detector in $\sqrt{s}=7 \mathrm{TeV} p p$, Phys. Rev. Lett. 106 (2011) 131802 [arXiv:1102.2357] [INSPIRE].

[112] CMS collaboration, V. Khachatryan et al., Search for supersymmetry in pp collisions at 7 $T e V$ in events with jets and missing transverse energy, Phys. Lett. B 698 (2011) 196 [arXiv: 1101.1628] [INSPIRE].

[113] ATLAS collaboration, G. Aad et al., Search for supersymmetry in pp collisions at $\sqrt{s}=7 \mathrm{TeV}$ in final states with missing transverse momentum and b-jets, Phys. Lett. B 701 (2011) 398 [arXiv: 1103.4344] [INSPIRE].

[114] CMS collaboration, S. Chatrchyan et al., Search for supersymmetry at the LHC in events with jets and missing transverse energy, arXiv:1109.2352 [INSPIRE].

[115] ATLAS collaboration, Search for squarks and gluinos using final states with jets and missing transverse momentum with the ATLAS detector in $\sqrt{s}=7 \mathrm{TeV}$ proton-proton collisions, ATLAS-CONF-2011-086 (2011).

[116] S. Akula, D. Feldman, Z. Liu, P. Nath and G. Peim, New constraints on dark matter from CMS and ATLAS data, Mod. Phys. Lett. A 26 (2011) 1521 [arXiv:1103.5061] [InSPIRE].

[117] S. Akula, D. Feldman, P. Nath and G. Peim, Excess observed in $C D F B_{s}^{0} \rightarrow \mu^{+} \mu^{-}$and SUSY at the LHC, arXiv:1107.3535 [INSPIRE].

[118] S. Akula et al., Interpreting the first CMS and ATLAS SUSY results, Phys. Lett. B 699 (2011) 377 [arXiv: 1103.1197] [INSPIRE].

[119] O. Buchmueller et al., Supersymmetry and dark matter in light of LHC 2010 and Xenon100 data, Eur. Phys. J. C 71 (2011) 1722 [arXiv:1106.2529] [INSPIRE].

[120] C. Boehm, A. Djouadi and M. Drees, Light scalar top quarks and supersymmetric dark matter, Phys. Rev. D 62 (2000) 035012 [hep-ph/9911496] [INSPIRE]. 
[121] Y. Santoso, Neutralino stop coannihilation in the CMSSM,

Nucl. Phys. Proc. Suppl. 124 (2003) 166 [hep-ph/0205026] [inSPIRE].

[122] P. Nath and R.L. Arnowitt, Predictions in SU(5) supergravity grand unification with proton stability and relic density constraints, Phys. Rev. Lett. 70 (1993) 3696 [hep-ph/9302318] [INSPIRE].

[123] K. Griest and D. Seckel, Three exceptions in the calculation of relic abundances, Phys. Rev. D 43 (1991) 3191 [inSPIRE].

[124] P. Gondolo and G. Gelmini, Cosmic abundances of stable particles: Improved analysis, Nucl. Phys. B 360 (1991) 145 [INSPIRE].

[125] E.J. Chun, J.-C. Park and S. Scopel, Dark matter and a new gauge boson through kinetic mixing, JHEP 02 (2011) 100 [arXiv: 1011.3300] [INSPIRE].

[126] P. Gondolo, P. Ko and Y. Omura, Light dark matter in leptophobic $Z^{\prime}$ models, arXiv:1106.0885 [INSPIRE].

[127] PAMELA collaboration, O. Adriani et al., An anomalous positron abundance in cosmic rays with energies 1.5-100 GeV, Nature 458 (2009) 607 [arXiv: 0810.4995] [INSPIRE].

[128] 2011 Fermi Symposium, May 9-12, Roma, Italy (2011), http://fermi.gsfc.nasa.gov/science/symposium/2011/.

[129] G. Angloher et al., Results from $730 \mathrm{~kg}$ days of the CRESST-II dark matter search, arXiv: 1109. 0702 [INSPIRE].

[130] CoGeNT collaboration, C. Aalseth et al., Results from a search for light-mass dark matter with a p-type point contact germanium detector, Phys. Rev. Lett. 106 (2011) 131301 [arXiv: 1002.4703] [INSPIRE].

[131] D. Feldman, Z. Liu and P. Nath, Low mass neutralino dark matter in the MSSM with constraints from $B_{s} \rightarrow \mu^{+} \mu^{-}$and Higgs search limits, Phys. Rev. D 81 (2010) 117701 [arXiv: 1003.0437] [INSPIRE].

[132] E. Kuflik, A. Pierce and K.M. Zurek, Light neutralinos with large scattering cross sections in the minimal supersymmetric standard model, Phys. Rev. D 81 (2010) 111701 [arXiv: 1003.0682] [INSPIRE].

[133] D.A. Vasquez, G. Bélanger, C. Boehm, A. Pukhov and J. Silk, Can neutralinos in the MSSM and NMSSM scenarios still be light?, Phys. Rev. D 82 (2010) 115027 [arXiv: 1009.4380] [INSPIRE].

[134] J.-J. Cao et al., Light dark matter in NMSSM and implication on Higgs phenomenology, Phys. Lett. B 703 (2011) 292 [arXiv:1104.1754] [INSPIRE].

[135] D.T. Cumberbatch, D.E. Lopez-Fogliani, L. Roszkowski, R.R. de Austri and Y.-L.S. Tsai, Is light neutralino as dark matter still viable?, arXiv:1107.1604 [INSPIRE].

[136] M. Vazques Acosta, Higgs searches at the LHC, talk given at the SUSY 2011, August 28-September 2, Fermilab, U.S.A. (2011).

[137] D. Feldman, K. Freese, P. Nath, B.D. Nelson and G. Peim, Predictive signatures of supersymmetry: measuring the dark matter mass and gluino mass with early LHC data, Phys. Rev. D 84 (2011) 015007 [arXiv:1102.2548] [INSPIRE].

[138] L.M. Krauss and F. Wilczek, Discrete gauge symmetry in continuum theories, Phys. Rev. Lett. 62 (1989) 1221 [INSPIRE]. 
[139] S.P. Martin, Implications of supersymmetric models with natural R-parity conservation, Phys. Rev. D 54 (1996) 2340 [hep-ph/9602349] [INSPIRE].

[140] G. Cacciapaglia, C. Csáki, G. Marandella and A. Strumia, The minimal set of electroweak precision parameters, Phys. Rev. D 74 (2006) 033011 [hep-ph/0604111] [INSPIRE].

[141] R. Barbier et al., R-parity violating supersymmetry, Phys. Rept. 420 (2005) 1 [hep-ph/0406039] [INSPIRE]. 